\title{
The Standardized Candle Method for Type li Plateau Supernovae
}

\section{Citation}

Olivares E., Felipe, Mario Hamuy, Giuliano Pignata, José Maza, Melina Bersten, Mark M. Phillips, Nicholas B. Suntzeff, et al. 2010. "THE STANDARDIZED CANDLE METHOD FOR TYPE II PLATEAU SUPERNOVAE." The Astrophysical Journal 715 (2): 833-53. https:// doi.org/10.1088/0004-637x/715/2/833.

\section{Permanent link}

http://nrs.harvard.edu/urn-3:HUL.InstRepos:41399764

\section{Terms of Use}

This article was downloaded from Harvard University's DASH repository, and is made available under the terms and conditions applicable to Other Posted Material, as set forth at http:// nrs.harvard.edu/urn-3:HUL.InstRepos:dash.current.terms-of-use\#LAA

\section{Share Your Story}

The Harvard community has made this article openly available.

Please share how this access benefits you. Submit a story.

Accessibility 


\title{
THE STANDARDIZED CANDLE METHOD FOR TYPE II PLATEAU SUPERNOVAE
}

\author{
Felipe Olivares E. ${ }^{1,2}$, Mario Hamuy ${ }^{1}$, Giuliano Pignata ${ }^{1,3}$, José Maza ${ }^{1}$, Melina Bersten ${ }^{1}$, Mark M. Phillips ${ }^{4}$, \\ Nicholas B. SuntzefF ${ }^{5}$, Alexei V. Filippenko ${ }^{6}$, Nidia I. Morrel ${ }^{4}$, Robert P. Kirshner ${ }^{7}$, and Thomas Matheson ${ }^{8}$ \\ ${ }^{1}$ Departamento de Astronomía, Universidad de Chile, Santiago, Chile \\ ${ }^{2}$ Max-Planck-Institut für Extraterrestrische Physik, 85740 Garching, Germany; felipe@ @mpe.mpg.de \\ ${ }^{3}$ Departamento de Ciencias Fisicas, Universidad Andres Bello, Avda. Republica 252, Santiago, Chile \\ ${ }^{4}$ Las Campanas Observatory, Carnegie Observatories, Casilla 601, La Serena, Chile \\ ${ }^{5}$ Physics Department, Texas A\&M University, College Station, TX 77843, USA \\ ${ }^{6}$ Department of Astronomy, University of California, Berkeley, CA 94720-3411, USA \\ ${ }^{7}$ Harvard-Smithsonian Center for Astrophysics, 60 Garden Street, Cambridge, MA 02138, USA \\ ${ }^{8}$ National Optical Astronomy Observatory, 950 North Cherry Avenue, Tucson, AZ 85719-4933, USA \\ Received 2009 November 15; accepted 2010 April 9; published 2010 May 4
}

\begin{abstract}
In this paper, we study the "standardized candle method" using a sample of 37 nearby (redshift $z<0.06$ ) Type II plateau supernovae having $B V R I$ photometry and optical spectroscopy. An analytic procedure is implemented to fit light curves, color curves, and velocity curves. We find that the $V-I$ color toward the end of the plateau can be used to estimate the host-galaxy reddening with a precision of $\sigma\left(A_{V}\right)=0.2 \mathrm{mag}$. The correlation between plateau luminosity and expansion velocity previously reported in the literature is recovered. Using this relation and assuming a standard reddening law $\left(R_{V}=3.1\right)$, we obtain Hubble diagrams (HDs) in the $B V I$ bands with dispersions of $\sim 0.4 \mathrm{mag}$. Allowing $R_{V}$ to vary and minimizing the spread in the HDs, we obtain a dispersion range of $0.25-0.30 \mathrm{mag}$, which implies that these objects can deliver relative distances with precisions of $12 \%-14 \%$. The resulting best-fit value of $R_{V}$ is $1.4 \pm 0.1$.
\end{abstract}

Key words: distance scale - galaxies: distances and redshifts - supernovae: general

Online-only material: color figures

\section{INTRODUCTION}

Core-collapse supernovae (CCSNe) are in general closely associated with star-forming regions in late-type galaxies (e.g., Anderson \& James 2008). Therefore, they have been attributed to stars born with initial mass $>8 M_{\odot}$ that undergo the collapse of their iron cores after a few million years of evolution and the subsequent ejection of their envelopes (e.g., Burrows 2000). According to theoretical studies, these SNe leave a compact object as a remnant, either a neutron star or a black hole (Baade \& Zwicky 1934; Arnett 1996; Janka et al. 2007).

Among CCSNe, we can observationally distinguish those with prominent hydrogen lines in their spectra (dubbed Type II; Minkowski 1941), those with no H but strong He lines (Type Ib), and those lacking $\mathrm{H}$ or He lines (Type Ic); see Filippenko (1997), and references therein. Although all of these objects are thought to share largely the same explosion mechanism, their different observational properties are explained in terms of how much of their H-rich and He-rich envelopes were retained prior to explosion. When the star explodes with a significant fraction of its initial H-rich envelope intact, it should display an H-rich spectrum and a light curve characterized by an optically thick phase of $\sim 100$ days of nearly constant luminosity called a "plateau," which comes to an end at the point when all of the hydrogen in the envelope has recombined. This phase is followed by a sudden drop of 2-3 mag and thereafter by an exponential decay in luminosity caused by the radioactive decay of ${ }^{56} \mathrm{Co}$ into ${ }^{56} \mathrm{Fe}$ (e.g., Nadyozhin 2003; Utrobin 2007). Nearly $50 \%$ of all CCSNe belong to this class of "Type II plateau" SNe (SNe IIP; Cappellaro et al. 1999; Botticella et al. 2008).

A decade ago, the standardized luminosities of Type Ia $\mathrm{SNe}$ (e.g., Phillips 1993; Hamuy et al. 1996; Riess et al. 1996; Phillips et al. 1999) led to the construction of Hubble diagrams (HDs) at redshifts $z=0-0.1$ with dispersions of only $\sim 0.15 \mathrm{mag}$, which opened up the opportunity of measuring the expected deceleration of the universe. At the end of the millennium, Riess et al. (1998) and Perlmutter et al. (1999) reported independent observations of high-redshift $\mathrm{SNe}(z=0.1-0.5)$ which permitted the measurement of the expansion history of the universe over $\sim 5 \mathrm{Gyr}$ of look-back time. Contrary to expectations, these observations revealed that the universe is currently described by an accelerated expansion. The discovery of cosmic acceleration, which was subsequently confirmed by additional studies of SNe Ia (e.g., Astier et al. 2006; Wood-Vasey et al. 2007; Hicken et al. 2009; Freedman et al. 2009), implies the possible existence of a cosmological constant, a concept initially introduced by Albert Einstein at the beginning of the 20th century. Its origin is still a mystery, and there are many other possible candidates for the repulsive "dark energy" that permeates the universe (see Frieman et al. 2008 for a review).

The acceleration of the universe has now been indirectly confirmed by other independent experiments such as the Wilkinson Microwave Anisotropy Probe (Spergel et al. 2007; Bennett et al. 2003) and baryon acoustic oscillations (Blake \& Glazebrook 2003; Seo \& Eisenstein 2003), as well as more directly with the integrated Sachs-Wolfe effect (e.g., Boughn \& Crittenden 2004; Fosalba et al. 2003) and X-ray observations of clusters of galaxies (Allen et al. 2008), but it is nevertheless important to obtain additional independent confirmation of the SN Ia results. Although SNe IIP are not as luminous and uniform as SNe Ia, Hamuy \& Pinto (2002) showed that the luminosities of the former can be standardized to levels of 0.4 and 0.3 mag in the $V$ and $I$ bands, respectively. This finding converted these objects into precise distance indicators and potentially into useful tools to measure cosmological parameters. Moreover, SNe IIP would be more abundant at high redshift than SNe Ia, since the star 
formation rate increases with redshift until $z \approx 2.5$ (see Hopkins $\&$ Beacom 2006, and references therein).

Type IIP SNe provide two routes to distance determinations. First, the "expanding photosphere method" (EPM; Kirshner \& Kwan 1974) is a technique based on theoretical models that is independent of the extragalactic distance scale (Eastman et al. 1996; Dessart \& Hillier 2005). In EPM the spectral energy distribution of the $\mathrm{SN}$ is approximated as a black body, and atmosphere models permit one to correct the dilution of the photospheric flux relative to a Planck function due to electron scattering. From a detailed analysis of the properties of the SN atmospheres, Eastman et al. (1996) showed that the most important variable in determining the dilution factors is the effective temperature. This result permits one to determine the photospheric angular radius of the SN from a measurement of the color temperature and infer the distance from the expansion velocity measured from spectral lines, without the need to craft specific models for each SN. This method can achieve dispersions of $0.3 \mathrm{mag}$ in the HD, which translates into a $14 \%$ precision in relative distances (Schmidt et al. 1994; Hamuy 2001; Jones et al. 2009). Another approach, more accurate than the latter, consists of adopting corrections and color temperatures from tailored models for each observation, revealing an accuracy of $\sim 10 \%$ in distance determination of individual SNe (Dessart \& Hillier 2006; Dessart et al. 2008; and the SEAM implementation of Baron et al. 2004).

Second, the "standardized candle method" (SCM) is an empirical technique initially proposed by Hamuy \& Pinto (2002) based on an observational correlation between the absolute magnitude of the $\mathrm{SN}$ and the expansion velocity of the photosphere, the luminosity/expansion-velocity (LEV) relation. This correlation shows that SNe IIP with greater luminosities have higher expansion velocities, which permits one to correct for the large ( $\sim 4 \mathrm{mag}$ ) luminosity differences displayed by these objects to levels of only $\sim 0.3 \mathrm{mag}$. So far, the SCM has been applied to 24 low-redshift $(z<0.05)$ SNe (Hamuy 2003b, 2005) and more recently by Poznanski et al. (2009) to 17 lowredshift $(z<0.03)$ SNe and by D'Andrea et al. (2010) to 15 intermediate-redshift $(z<0.144)$, as well as by Nugent et al. (2006) to 5 high-redshift $(z<0.29)$ SNe. The latter work was the first attempt to derive cosmological parameters from $\mathrm{SNe}$ IIP and demonstrated the enormous potential of this class of objects as cosmological probes. A similar attempt to standardize the luminosities of SNe IIP was done by Elmhamdi et al. (2003) based on the steepness of the $V$ light-curve slope at the inflection time.

Several major sky-survey programs now in progress or to be deployed in the upcoming years, such as the Palomar Transient Factory (Rau et al. 2009; Law et al. 2009), the Panoramic Survey Telescope and Rapid Response System (Hodapp et al. 2004), the Large Synoptic Survey Telescope (Tyson et al. 2003), the Visible and Infrared Survey Telescope (Emerson et al. 2004), the VLT Survey Telescope (Capaccioli et al. 2003), the Dark Energy Survey (Castander 2007), and the SkyMapper (Granlund et al. 2006), offer the promise of discovering $\mathrm{SNe}$ by the thousands. Thus, we will be inevitably confronted by enormous amounts of data on SNe IIP which will contain potentially valuable cosmological information. The challenge is to find an implementation of SCM that does not require spectroscopic data since these surveys will be purely photometric.

In spite of the great potential shown by the SCM, it still suffers from a variety of problems which need to be addressed: (1) the lack of a well-defined maximum in the light curves has prevented us from defining the phase of each event; (2) each
SN shows a different color evolution, which has compromised the use of the photometric data for the determination of hostgalaxy extinction; (3) the assumption for dereddening employed by Hamuy \& Pinto (2002), namely, that all SNe reach the same asymptotic color toward the end of the plateau phase, has often led to negative extinction corrections, so it still needs to be further tested; and (4) the small sample size used so far, especially the scarcity of SNe in the Hubble flow, has prevented a proper determination of the intrinsic precision of the method.

With these obstacles in mind, we have developed a robust mathematical procedure to fit the light curves, color curves, and velocity curves, in order to obtain a more accurate determination of the relevant parameters required by the SCM (magnitudes, colors, and ejecta velocities), as described in detail in the thesis work of Olivares (2008). Here, we apply this technique to 37 SNe IIP in order to construct an HD, evaluate the accuracy of the SCM, and obtain an independent determination of the Hubble constant. Since our sample has several objects in common with the recent EPM analysis of Jones et al. (2009), we perform a comparison between SCM and EPM.

We organize this paper as follows. In Section 2, we describe all of our observational material including telescopes, instruments, and surveys involved. The analysis, methodology, and procedures, such as the $A_{\mathrm{G}}, K$, and $A_{\text {host }}$ corrections, are explained in detail in Section 3. The dereddening analysis, the $\mathrm{HD}$, the value of the Hubble constant, and the comparison between SCM and EPM distances are addressed in Section 4. The final remarks in Section 5 provide a discussion about variations of the reddening law for SNe IIP and the possibility of a nonstandard extinction law in the SN host galaxies. We present our conclusions in Section 6.

\section{OBSERVATIONAL MATERIAL}

This work makes use of data obtained in the course of four systematic $\mathrm{SN}$ follow-up programs carried out during the years 1986-2003: (1) the Cerro Tololo SN program (1986-1996); (2) the Calán/Tololo SN program (CT, 1990-1993); (3) the Optical and Infrared Supernova Survey (SOIRS, 1999-2000); and (4) the Carnegie Type II Supernova Program (CATS, 2002-2003). As a result of these efforts, photometry and spectroscopy (some infrared but mostly optical) was obtained for nearly $100 \mathrm{SNe}$ of all types, 51 of which belong to the Type II class. Of these 51 SNe II, a subset of 33 objects meets the requirements for this study. Next, we describe in general terms the data acquisition and reduction procedures. For more details the reader can refer to M. Hamuy et al. (2010, in preparation).

\subsection{Photometric Data}

The photometry was acquired with telescopes from Cerro Tololo Inter-American Observatory (CTIO), Las Campanas Observatory, the European Southern Observatory (ESO) in La Silla, and Steward Observatory. Many different telescopes and instruments were used to generate this data set as shown in Table 1. In all cases, we employed CCD detectors and standard Johnson-Kron-Cousins-Hamuy UBVRIZ filters (Johnson et al. 1966; Cousins 1971; Hamuy et al. 2001).

The images were processed with IRAF $^{9}$ through bias subtraction and flat-fielding. All of them were further processed through the step of galaxy subtraction using template images of the host

\footnotetext{
9 IRAF is distributed by the National Optical Astronomy Observatory, which is operated by the Association of Universities for Research in Astronomy, Inc., under cooperative agreement with the National Science Foundation; see http://iraf.noao.edu.
} 
Table 1

Telescopes and Instruments

\begin{tabular}{lcc}
\hline \hline \multicolumn{1}{c}{ Telescope } & Instrument & Phot $/ \mathrm{Spec}$ \\
\hline CTIO $0.9 \mathrm{~m}$ & CCD & $\mathrm{P}$ \\
YALO $1.0 \mathrm{~m}$ & ANDICAM & $\mathrm{P}$ \\
YALO $1.0 \mathrm{~m}$ & 2DF & $\mathrm{S}$ \\
CTIO $1.5 \mathrm{~m}$ & CCD & $\mathrm{P}$ \\
CTIO $1.5 \mathrm{~m}$ & CSPEC & $\mathrm{S}$ \\
Blanco $4.0 \mathrm{~m}$ & CSPEC & $\mathrm{S}$ \\
Blanco $4.0 \mathrm{~m}$ & 2DF & $\mathrm{S}$ \\
Blanco $4.0 \mathrm{~m}$ & CCD & $\mathrm{P}$ \\
Swope $1.0 \mathrm{~m}$ & CCD & $\mathrm{P}$ \\
du Pont $2.5 \mathrm{~m}$ & WFCCD & $\mathrm{P} / \mathrm{S}$ \\
du Pont $2.5 \mathrm{~m}$ & MODSPEC & $\mathrm{S}$ \\
du Pont $2.5 \mathrm{~m}$ & 2DF & $\mathrm{S}$ \\
du Pont $2.5 \mathrm{~m}$ & CCD & $\mathrm{P}$ \\
Baade $6.5 \mathrm{~m}$ & LDSS2 & $\mathrm{P} / \mathrm{S}$ \\
Baade $6.5 \mathrm{~m}$ & B\&C & $\mathrm{S}$ \\
Clay $6.5 \mathrm{~m}$ & LDSS2 & $\mathrm{P} / \mathrm{S}$ \\
ESO $1.5 \mathrm{~m}$ & IDS & $\mathrm{S}$ \\
Danish $1.5 \mathrm{~m}$ & DFOSC & $\mathrm{P} / \mathrm{S}$ \\
MPIESO $2.2 \mathrm{~m}$ & EFOSC2 & $\mathrm{S}$ \\
NTT $3.6 \mathrm{~m}$ & EMMI & $\mathrm{S}$ \\
ESO $3.6 \mathrm{~m}$ & EFOSC & $\mathrm{S}$ \\
Kuiper $1.5 \mathrm{~m}$ & CCD & $\mathrm{P}$ \\
Bok $2.2 \mathrm{~m}$ & B\&C & $\mathrm{S}$ \\
Shane $3.0 \mathrm{~m}$ & Kast & $\mathrm{S}$ \\
Whipple $1.5 \mathrm{~m}$ & FAST & $\mathrm{S}$ \\
\hline
\end{tabular}

Note. Whether the instrument was used for photometry $(\mathrm{P})$, spectroscopy $(\mathrm{S})$, or both $(\mathrm{P} / \mathrm{S})$ is listed in Column 3.

galaxies. Photometric sequences were established around each SN based on observations of Landolt and Hamuy standards (Landolt 1992; Hamuy et al. 1992, 1994). The photometry of all $\mathrm{SNe}$ was performed differentially with respect to the local sequence on the galaxy-subtracted images. The transformation of instrumental magnitudes into the standard system was done by taking into account a linear color term and a zero point. Although this procedure partially removes the instrument-toinstrument differences in the SN magnitudes, it should be kept in mind that significant systematic discrepancies can still remain owing to the nonstellar nature of the SN spectrum (e.g., Hamuy et al. 1990).

Complementary photometry for SN 2003gd obtained by Van Dyk et al. (2003) and Hendry et al. (2005) was also incorporated in our analysis.

\subsection{Spectroscopic Data}

The spectroscopic data were also obtained with a great variety of instruments and telescopes, as shown in Table 1. The observations consisted of the SN observation immediately followed by an emission-line comparison lamp taken at the same position in the sky, and 2-3 flux standards per night from the list of Hamuy et al. (1992, 1994). We always used CCD detectors, in combination with different gratings/grisms and blocking filters. The reductions were performed with IRAF and consisted of bias subtraction, flat-fielding, one-dimensional spectrum extraction and sky subtraction, wavelength calibration, and flux calibration. No attempts were made to remove the telluric lines.

Our spectroscopic database was complemented with spectra obtained by the CfA SN program with the FAST CCD spectrograph (Fabricant et al. 1998) at the $1.5 \mathrm{~m}$ telescope of the Whipple Observatory (see Matheson et al. 2008 for details on the data reduction), and by the University of California,
Berkeley SN program with the Kast double CCD spectrograph (Miller \& Stone 1993) on the Shane $3 \mathrm{~m}$ telescope at Lick Observatory. The above standard procedures, including removal of telluric absorption lines, were employed to reduce the Lick spectra (e.g., Foley et al. 2003).

\subsection{Subsample Used for this Work}

Of the 51 SNe II observed in the course of these four surveys, a subset of 33 objects comply with the requirements of (1) being a member of the plateau subclass; (2) having sufficient spectroscopic temporal coverage; and (3) having light curves with good temporal coverage. To this sample we added four SNe from the literature: SN 1999gi, SN 2004dj, SN 2004et, and SN 2005cs. Table 2 lists our final sample of 37 SNe IIP. For each SN this table includes the name of the host galaxy, the equatorial coordinates of the $\mathrm{SN}$, the heliocentric redshift and its source, the reddening due to our own Galaxy (Schlegel et al. 1998), and the survey during which the SN was observed.

\section{METHODOLOGY AND PROCEDURES}

In the thesis work of Olivares (2008), we describe in detail the techniques for correcting the photometry for Galactic absorption $\left(A_{\mathrm{G}}\right), K$-correction terms, and host-galaxy dust absorption $\left(A_{\text {host }}\right)$ - the so-called " $A K A$ corrections." In brief, we employ a library of 196 spectra of SNe II (including other SN II types than just $\mathrm{SNe}$ IIP) to synthesize $K$-terms and absorption coefficients given the specific redshift and $A_{V}$ value of each SN. Synthetic $B-V$ and $V-I$ colors are used as proxies for the spectral energy distribution in order to account for the evolution of each SN.

\subsection{Fits to Light, Color, and Velocity Curves}

In the first incarnation of the SCM, Hamuy \& Pinto (2002) measured all of the relevant quantities (magnitudes, colors, and velocities) at fixed epochs with respect to the time of explosion. In most cases, however, it proves hard to constrain this time, thus hampering the task to compare data obtained for different $\mathrm{SNe}$. It would be ideal to have a conspicuous feature in the light curves; unfortunately, unlike other SN types, SNe IIP generally do not show a well-defined maximum during the plateau phase.

One way around this is to use the end of the plateau as an estimate of the time origin for each event. In practice, it is not easy to measure this time owing to the generally coarser sampling of the light curves at this phase. Thus, our first aim is to implement a robust light-curve fitting procedure in order to obtain a reliable time origin to be used as a uniform reference epoch to measure magnitudes, colors, and expansion velocities. In the remainder of this section, we proceed to implement fitting methods to measure reliable colors and expansion velocities.

\subsubsection{BVRI Light-curve Fits}

Figure 1 shows the BVRI light curves of SN 1999em. As can be seen, there are three distinguishable phases in the light curves.

1. The plateau phase, in which the SN shows an almost constant luminosity during the first $\sim 100$ days of its evolution. This phase corresponds to the optically thick period in which a hydrogen recombination wave recedes in mass coordinate, gradually releasing the internal energy of the star (see Kirshner \& Kwan 1974; Nadyozhin 2003; Utrobin 2007; Bersten \& Hamuy 2009, and references therein).

2. The linear or radioactive tail, a linear decay in magnitude (exponential in flux) starting about 130 days after the 
Table 2

SN Sample

\begin{tabular}{|c|c|c|c|c|c|c|c|}
\hline SN Name & Host Galaxy & $\begin{array}{c}\alpha(\mathrm{J} 2000) \\
(\mathrm{h} \mathrm{m} \mathrm{s})\end{array}$ & $\begin{array}{c}\delta(\mathrm{J} 2000) \\
\left({ }^{\circ} \prime \prime \prime\right)\end{array}$ & $z_{\text {host }^{a}}{ }^{a}$ & $(s)^{b}$ & $\begin{array}{c}E(B-V)_{\mathrm{Gall}}^{\mathrm{c}} \\
(\mathrm{mag})\end{array}$ & References \\
\hline 1991al & LEDA 140858 & 194224.00 & -550623.0 & 0.01525 & HP02 & 0.051 & 1 \\
\hline 1992af & ESO 340-G038 & 203040.20 & -421835.0 & 0.01847 & NED & 0.052 & 1 \\
\hline 1992am & MCG-01-04-039 & 012502.70 & -043901.0 & 0.04773 & NED & 0.049 & 1 \\
\hline 1992ba & NGC 2082 & 054147.10 & -641801.0 & 0.00395 & NED & 0.058 & 1 \\
\hline 1993A & Anonymous & 073917.30 & -620314.0 & 0.02800 & NED & 0.173 & 1 \\
\hline $1999 \mathrm{br}$ & NGC 4900 & 130041.80 & +022946.0 & 0.00320 & NED & 0.024 & 2 \\
\hline $1999 \mathrm{ca}$ & NGC 3120 & 100522.90 & -341241.0 & 0.00931 & NED & 0.109 & 2 \\
\hline $1999 \mathrm{cr}$ & ESO 576-G034 & 132018.30 & -200850.0 & 0.02020 & NED & 0.098 & 2 \\
\hline $1999 \mathrm{em}$ & NGC 1637 & 044127.04 & -025145.2 & 0.00267 & NED & 0.040 & 2 \\
\hline 1999gi & NGC 3184 & 101817.00 & +412528.0 & 0.00198 & NED & 0.017 & 3 \\
\hline 0210 & MCG +00-03-054 & 010116.80 & -010552.0 & 0.05140 & NED & 0.036 & 4 \\
\hline $2002 \mathrm{fa}$ & NEAT J205221.51 & 205221.80 & +020842.0 & 0.06000 & NED & 0.099 & 4 \\
\hline $2002 \mathrm{gw}$ & NGC 922 & 022502.97 & -244750.6 & 0.01028 & NED & 0.020 & 4 \\
\hline $2002 \mathrm{hj}$ & NPM1G +04.0097 & 025809.30 & +04 4104.0 & 0.02360 & NED & 0.115 & 4 \\
\hline $2002 h x$ & PGC 23727 & 082739.43 & -144715.7 & 0.03099 & NED & 0.054 & 4 \\
\hline 2003B & NGC 1097 & 024613.78 & -301345.1 & 0.00424 & NED & 0.027 & 4 \\
\hline $2003 E$ & MCG-4-12-004 & 043910.88 & -241036.5 & 0.01490 & J09 & 0.048 & 4 \\
\hline $2003 \mathrm{~T}$ & UGC 4864 & 091411.06 & +164448.0 & 0.02791 & NED & 0.031 & 4 \\
\hline 2003bl & NGC 5374 & 135730.65 & +060536.4 & 0.01459 & J09 & 0.027 & 4 \\
\hline $2003 b n$ & 2MASX J10023529 & 100235.51 & -211054.5 & 0.01277 & NED & 0.065 & 4 \\
\hline $2003 \mathrm{ci}$ & UGC 6212 & 111023.83 & +04 4935.9 & 0.03037 & NED & 0.060 & 4 \\
\hline $2003 \mathrm{cn}$ & IC 849 & 130737.05 & -005649.9 & 0.01811 & J09 & 0.021 & 4 \\
\hline $2003 c x$ & NEAT J135706.53 & 135706.46 & -170222.6 & 0.03700 & NED & 0.094 & 4 \\
\hline $2003 \mathrm{ef}$ & NGC 4708 & 124942.25 & -110529.5 & 0.01480 & J09 & 0.046 & 4 \\
\hline $2003 \mathrm{fb}$ & UGC 11522 & 201150.33 & +054537.6 & 0.01754 & J09 & 0.183 & 4 \\
\hline $2003 g d$ & M74 & 013642.65 & +154420.9 & 0.00219 & NED & 0.069 & $4,5,6$ \\
\hline 2003hd & MCG-04-05-010 & 014946.31 & -215437.8 & 0.03950 & NED & 0.013 & 4 \\
\hline 2003hg & NGC 7771 & 235124.13 & +200638.3 & 0.01427 & NED & 0.074 & 4 \\
\hline $2003 \mathrm{hk}$ & NGC 1085 & 024625.76 & +033632.2 & 0.02265 & NED & 0.037 & 4 \\
\hline 2003hl & NGC 772 & 015921.28 & +190014.5 & 0.00825 & NED & 0.073 & 4 \\
\hline $2003 \mathrm{hn}$ & NGC 1448 & 034436.10 & -443749.0 & 0.00390 & NED & 0.014 & 4 \\
\hline 2003ho & ESO 235-G58 & 210630.56 & -480729.9 & 0.01438 & NED & 0.039 & 4 \\
\hline 2003ip & UGC 327 & 003315.40 & +075418.0 & 0.01801 & NED & 0.066 & 4 \\
\hline $2003 i q$ & NGC 772 & 015919.96 & +185942.1 & 0.00825 & NED & 0.073 & 4 \\
\hline 2004dj & NGC 2403 & 073717.00 & +653558.1 & 0.00044 & NED & 0.040 & 7 \\
\hline $2004 \mathrm{et}$ & NGC 6946 & 203525.30 & +600718.0 & 0.00016 & NED & 0.342 & 8 \\
\hline $2005 \mathrm{cs}$ & NGC 5194 & 132953.40 & +471028.0 & 0.00154 & NED & 0.035 & 9,10 \\
\hline
\end{tabular}

Notes.

${ }^{a}$ Heliocentric host-galaxy redshift.

b Source of host-galaxy redshift—HP02: Hamuy \& Pinto (2002); J09: Jones et al. (2009); NED: NASA/IPAC Extragalactic Database.

${ }^{c}$ Galactic reddenings were obtained from Schlegel et al. (1998).

References. (1) Calán/Tololo Supernova Program; (2) SOIRS; (3) Leonard et al. 2002b; (4) Carnegie Type II Supernova Survey (CATS); (5) Van Dyk et al. 2003; (6) Hendry et al. 2005; (7) Vinkó et al. 2006; (8) Sahu et al. 2006; (9) Pastorello et al. 2006; (10) Tsvetkov et al. 2006.

explosion. This phase corresponds to the optically thin period powered by the ${ }^{56} \mathrm{Co} \rightarrow{ }^{56} \mathrm{Fe}$ radioactive decay (e.g., Weaver \& Woosley 1980).

3. A transition phase of $\sim 30$ days between the plateau and linear phases.

Both the plateau and linear phases are trivial to model if taken separately, but the abrupt transition makes the fitting task much more challenging, especially with coarsely sampled light curves. After considering several options we ended up using the arithmetic sum of the three functions shown in Figure 1, as follows.

1. A Fermi-Dirac function (dotted line in Figure 1), which provides a very good description of the transition between the plateau and radioactive phases:

$$
f_{\mathrm{FD}}(t)=\frac{-a_{0}}{1+e^{\left(t-t_{\mathrm{PT}}\right) / w_{0}}},
$$

where $a_{0}$ represents the height of the step in units of magnitude, $t_{\mathrm{PT}}$ corresponds to the middle of the transition phase and is a natural candidate to define the origin of the time axis, and $w_{0}$ quantifies the width of the transition phase. At $t=t_{\mathrm{PT}}-3 w_{0}$ the height of the step has been reduced by $4.7 \%$, and it decreases down to $95.3 \%$ at $t=t_{\mathrm{PT}}+3 w_{0}$.

2. A straight line (long-dashed line in Figure 1) which accounts for the slope due to the radioactive decay:

$$
l(t)=p_{0}\left(t-t_{\mathrm{PT}}\right)+m_{0},
$$

where $p_{0}$ corresponds to the slope of radioactive tail and an approximate slope for the plateau in units of mag per day, and $m_{0}$ corresponds to the zero point in magnitude at $t=t_{\mathrm{PT}}$.

3. A Gaussian function (short-dashed line in Figure 1), which serves mainly for fitting the $B$ light-curve bump and the $I$ 


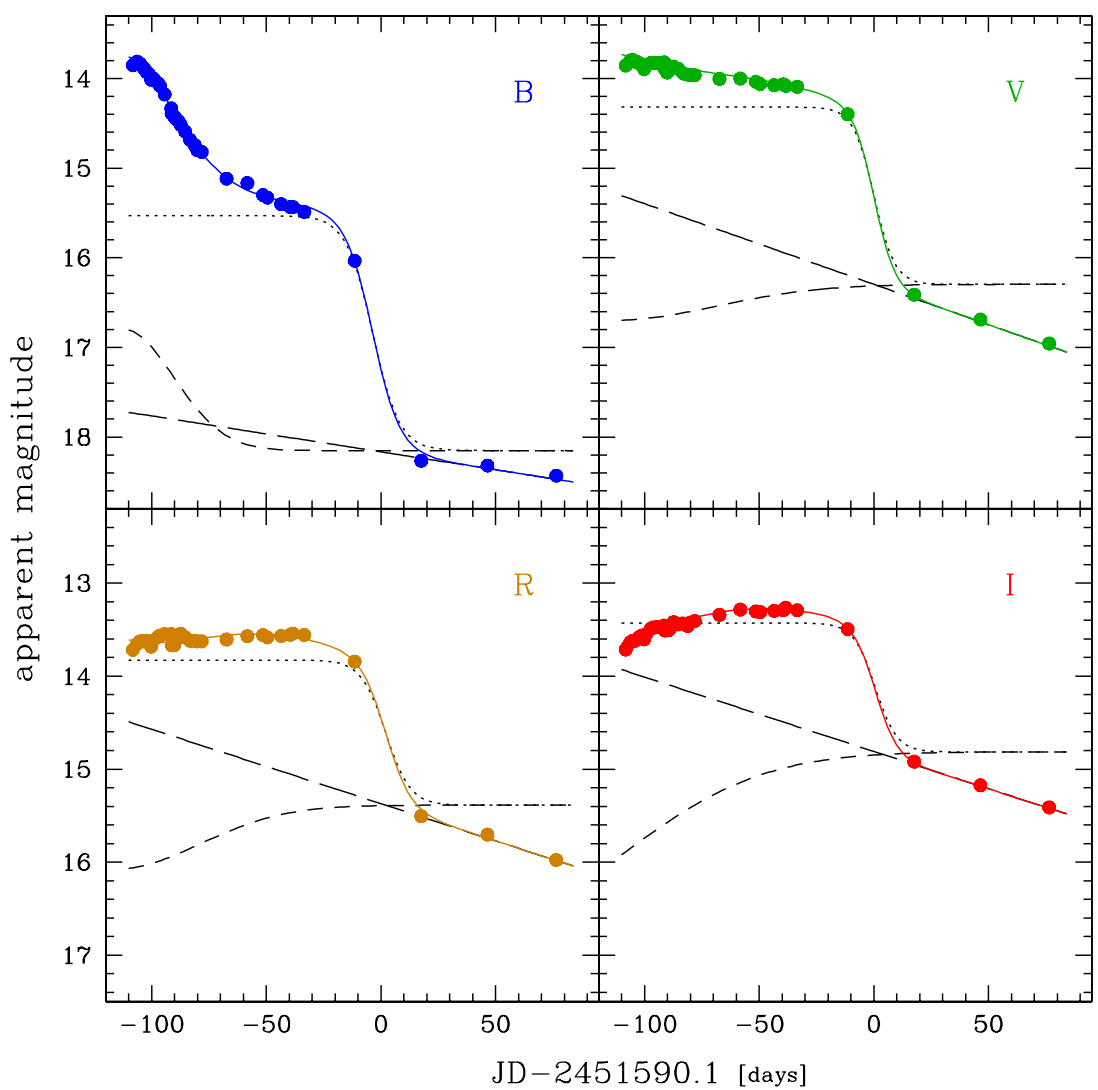

Figure 1. BVRI light curves of SN 1999em. BVRI magnitudes are respectively shown in top left, top right, bottom left, and bottom right panels. The solid line corresponds to the analytic fit. The segmented lines represent the decomposition of the main function into its three components: the Fermi-Dirac function (dotted line), a straight line (long-dashed line), and a Gaussian function (short-dashed line). The origin of the time axis corresponds to $t_{\mathrm{PT}}$ in Julian days for SN $1999 \mathrm{em}$. Since the instrumental error bars are smaller than the symbols, they are not shown.

(A color version of this figure is available in the online journal.)

light-curve curvature during the plateau phase:

$$
g(t)=-P e^{-\left(\frac{t-Q}{R}\right)^{2}},
$$

where $P$ is the height of the Gaussian peak in units of magnitudes, $Q$ is the center of the Gaussian function in days, and $R$ is the width of the Gaussian function. The Gaussian function is also useful for reproducing the smallscale features that can appear in the $V$-band plateau.

The resulting analytic function we use to model the light curves is the sum of the three functions detailed above,

$$
\begin{aligned}
F(t) & =f_{\mathrm{FD}}(t)+l(t)+g(t) \\
& =\frac{-a_{0}}{1+e^{\left(t-t_{\mathrm{PT}}\right) / w_{0}}}+p_{0}\left(t-t_{\mathrm{PT}}\right)+m_{0}-P e^{-\left(\frac{t-Q}{R}\right)^{2}},
\end{aligned}
$$

which has eight free parameters. This function is fitted to the individual light curves in unison (not by parts) using a $\chi^{2}$ minimizing procedure. In order to find the minimum $\chi^{2}$, we use the Downhill Simplex Method (Nelder \& Mead 1965). Despite being not very efficient in terms of the number of iterations required, it provides robust solutions. Examples of the analytic fits are shown as solid lines in Figure 1. Although we cannot model most of the small-scale features in the plateau, the fitting does a very good job in modeling the transition. Furthermore, the analytic function gives us important parameters that characterize the light-curve shape, particularly $t_{\mathrm{PT}}$ which provides a time origin. In the plots in Figure 1, the time axis is chosen to coincide with the value of this parameter obtained from the $V$ light curve. In Table 3 , we gather the eight parameters and the corresponding reduced $\chi^{2}\left(\chi_{v}^{2}\right)$ of the $V$ light curve fitting for the whole SN sample.

A critical quantity in the analysis that follows is $t_{\mathrm{PT}}$ and its uncertainty, both of which determine the uncertainties in all of the relevant SCM quantities. For most cases, when the tail phase has been observed, the $\chi^{2}$ minimizing routine has no difficulties finding $t_{\mathrm{PT}}$ and delivers a credible error. On the other hand, when the light curve does not have any late-time data points, the routine underestimates $\sigma\left(t_{\mathrm{PT}}\right)$, in which case we need to provide a more realistic estimate of this uncertainty. Only for a few cases did we have to arbitrarily set $t_{\mathrm{PT}}$ and its error based on the light-curve sampling (see Column 3 in Table 3).

Regardless of the sampling of the light curves, we assign a minimum of $\sigma\left(t_{\mathrm{PT}}\right)=2$ days. Although these uncertainties in $t_{\text {PT }}$ are very conservative and arbitrary, they do not translate into high or unrealistic errors in quantities such as magnitudes, colors, or expansion velocities, as we will see later.

Note that Elmhamdi et al. (2003) used a similar approach as the one described here, except that their model function only has five independent parameters and is somewhat simpler than ours.

\subsubsection{Color-curve Fits}

Figure 2 shows the $B-V, V-R$, and $V-I$ colors of three prototypical SNe IIP corrected for $A_{\mathrm{G}}$ and $K$-terms as described by Olivares (2008, chapter 3.1). The time origin in the abscissa corresponds to $t_{\mathrm{PT}}$, the middle of the transition phase. In each case we employ all the data points between days -100 and -10 
Table 3

Parameters from the Analytical Fitting to the $V$ Light Curve

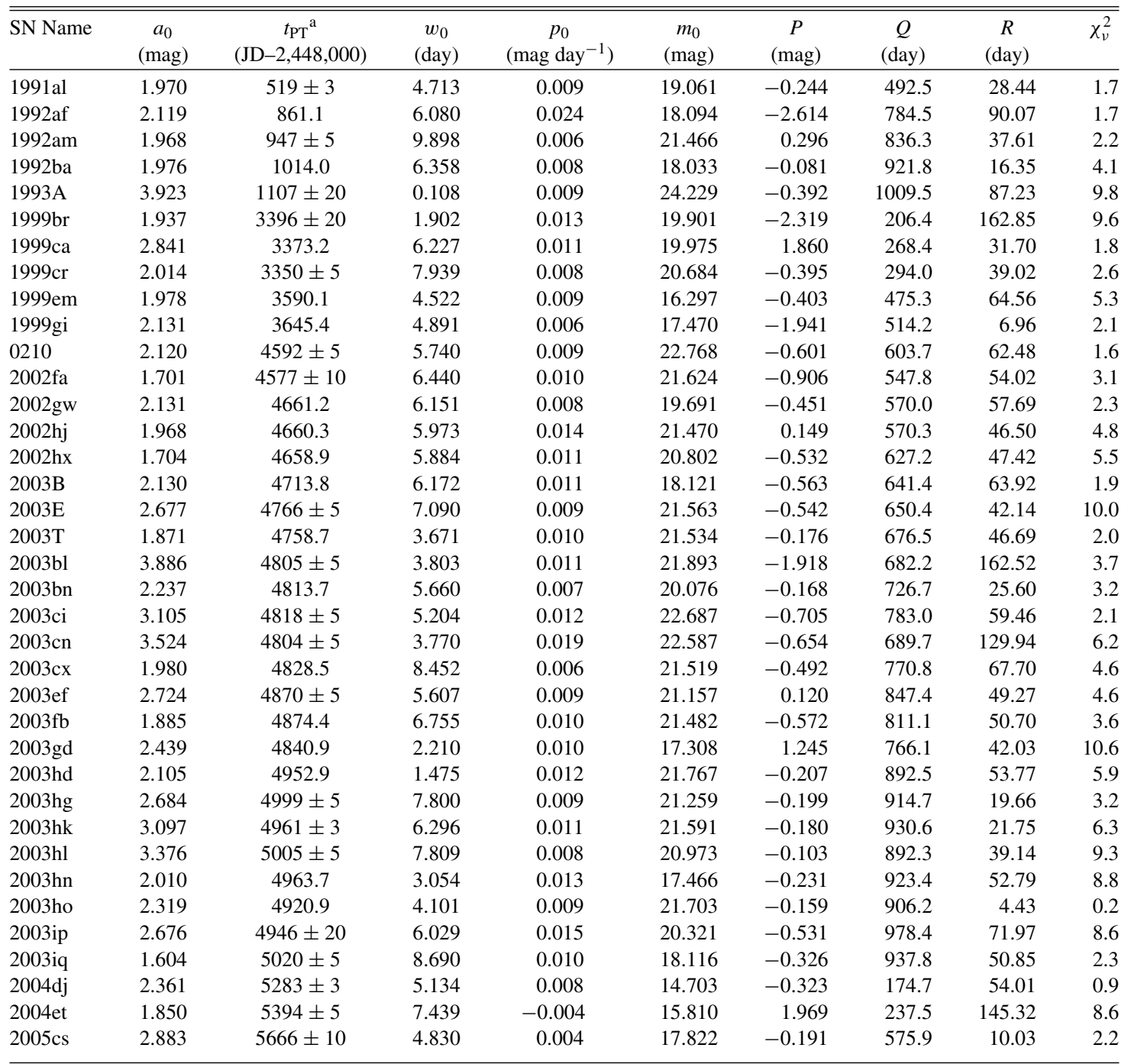

Note. ${ }^{\text {a }}$ The minimum uncertainty of $t_{\mathrm{PT}}$ is set to a conservative value of 2 days and is assigned to those SNe for which an uncertainty is not quoted. For three cases $t_{\mathrm{PT}}$ is manually determined with an uncertainty of 20 days.

to fit a Legendre polynomial shown with solid lines in Figure 2. The degree of the polynomial was chosen on a case-by-case basis and varied between third and sixth order. It is evident that, during the plateau phase, the photosphere gets redder with time owing to the decrease of the surface temperature as the $\mathrm{SN}$ expands and to a redistribution of flux from blue to red wavelengths due to line blanketing. According to the theory, the photospheric temperature should approach and never get below the temperature of hydrogen recombination around $5000 \mathrm{~K}$. Based on this physical argument, Hamuy \& Pinto (2002) argued that all SNe IIP should reach the same intrinsic colors toward the end of the plateau phase; hence, they proposed that the color excesses measured at this phase could be attributed to dust reddening in the $\mathrm{SN}$ host galaxy and be exploited to measure $A_{\text {host }}$. Hamuy \& Pinto (2002) performed their analysis with a simple naked-eye estimate of the asymptotic colors. Here, we improve significantly this situation through the formal colorcurve fitting procedure described above.

Armed with the polynomial fits, we proceeded to interpolate colors on a continuous grid with one-day spacing between days -80 and -10 for analyzing colors at multiple epochs (see Section 3.2). In a handful of cases, the data did not encompass the whole grid and we had to extrapolate colors, but never by more than 3 days from the nearest data point.

\subsubsection{Fe II Based Expansion-velocity Curves}

The third ingredient for SCM is the velocity of the SN ejecta. Different spectral lines yield different expansion velocities depending on the region from which the photon is emitted. The Fe II $\lambda 5169$ line is thought to be a good proxy for SN IIP photospheric velocity (Dessart \& Hillier 2005) and has been previously employed for SCM. Here, we use the Fe II expansion velocities measured by Jones et al. (2009) from the minimum of the P Cygni line profile after correcting the spectra for the heliocentric redshifts of the host galaxies. The uncertainties of these measurements were estimated to be $\sim 85 \mathrm{~km} \mathrm{~s}^{-1}$ from the quality of the wavelength calibration and the signal-tonoise ratio $(\mathrm{S} / \mathrm{N})$ of the absorption feature of the $\mathrm{P}$ Cygni line profile. Although these measurements do not consider relativistic corrections, the latter prove negligible $\left(\lesssim 40 \mathrm{~km} \mathrm{~s}^{-1}\right)$ in the range considered here $\left(2000-5000 \mathrm{~km} \mathrm{~s}^{-1}\right)$.

Figure 3 shows Fe II velocities as a function of SN phase, for four different $\mathrm{SNe}$ selected for their wide range of sampling 


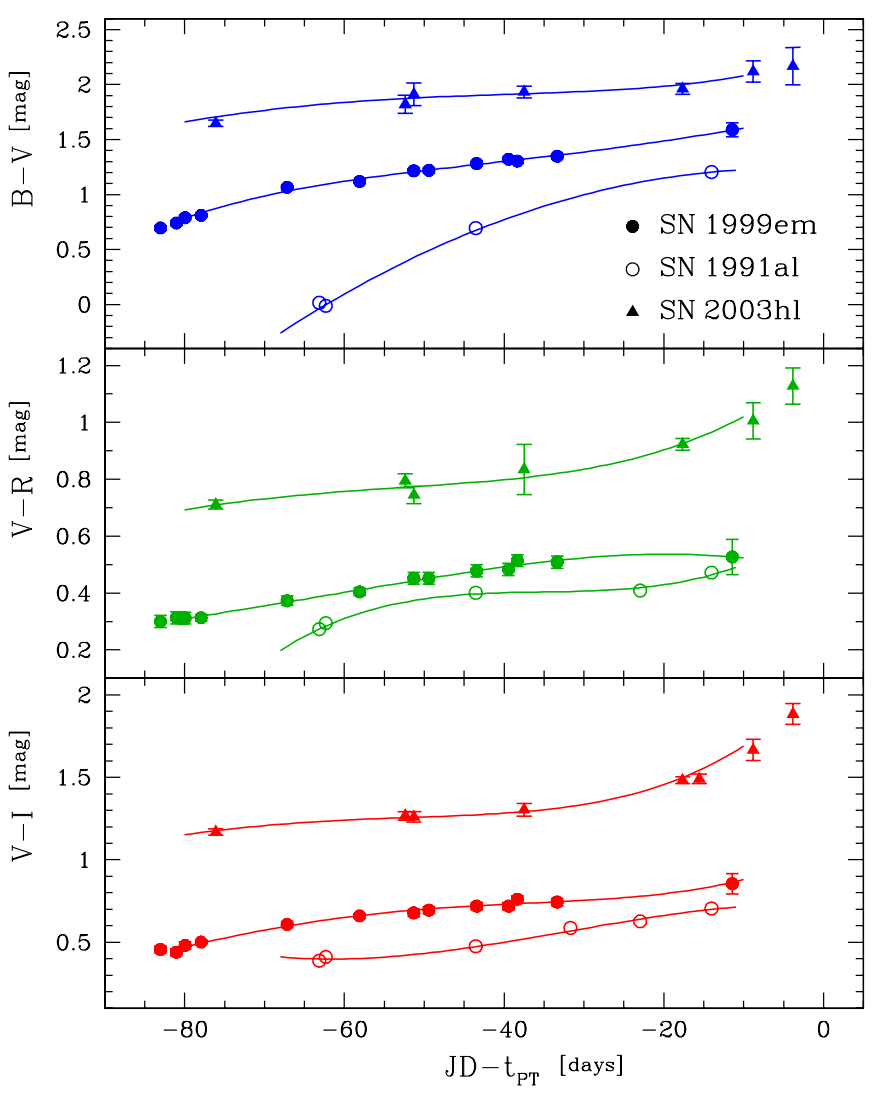

Figure 2. $B-V, V-R$, and $V-I$ color curves of $\mathrm{SN} 1999 \mathrm{em}$ (filled circles), SN 1991al (open circles), and SN 2003hl (filled triangles) corrected for the $A_{\mathrm{G}}$ and $K$-terms. This comparison demonstrates that each SN displays a different color evolution, precluding the determination of color excesses from a simple color offset after correcting for the $A_{\mathrm{G}}$ and $K$-terms. For SN 1991al, the error bars are always smaller than the symbols and therefore not plotted.

(A color version of this figure is available in the online journal.)

characteristics. As shown by Hamuy \& Pinto (2002), the Fe II $\lambda 5169$ expansion velocity curve during the plateau phase can be properly modeled with a power law, which we choose to be of the form

$$
v_{\mathrm{FeII}}(t)=A\left(t-t_{0}\right)^{\alpha},
$$

where $A, t_{0}$, and $\alpha$ are free fitting parameters without obvious physical meaning.

In general, we fit for all three parameters, but when only two velocity measurements are available (e.g., SN 1992af in the top left panel of Figure 3) we fix the $\alpha$ exponent to -0.5 , which corresponds to a typical value for our sample. As shown with solid lines in Figure 3, the fits are quite satisfactory. Here, we choose to restrict the power-law fits to the plateau phase, since the power-law behavior is not observed for expansion velocities beyond the transition phase. We use the same one-day continuous grid as for the color curves in order to interpolate velocities at different epochs between -80 and -10 days. Given the good quality of the fits and the shallow slope at late epochs, we allow extrapolations of up to 15 days past the nearest data point (see SN 1999cr in Figure 3). At the early-time boundary, we reduce the extrapolations to 10 days prior to the first point, because the power law becomes steeper at early times (e.g., see SN 2003gd in Figure 3). When only two velocity measurements are available, we reduce the extrapolations by 5 days. Since at the end of the plateau phase the SN atmosphere starts to become nebular, we discarded data points beyond the transition in the velocity fits. We do not impose any constraints on the first data point.

\subsection{Host-galaxy Extinction Determination}

To determine the host-galaxy extinction, we assume that all SNe IIP should evolve from a hot initial stage to one of uniform photospheric temperature, owing to the hydrogenrecombination nature of their photospheres.

Although simple in theory, there are a few practical difficulties when comparing the color evolution. First, it is not always possible to constrain the time of explosion and accurately compare color curves of different objects. One way around this is to use the transition time, $t_{\mathrm{PT}}$, defined in Section 3.1.1. The second problem is illustrated in Figure 2: the color-curve shapes can vary significantly from one $\mathrm{SN}$ to another, preventing one from measuring a single color offset between two SNe. To circumvent this, we pick a single fiducial epoch in the color evolution and assess the performance of the color as a reddening estimator. Our polynomial fits to the color curves are very convenient for this purpose, since they allow us to interpolate reliable colors on a day-to-day basis over a wide range of epochs and explore which epoch gives the best results.

If all SNe share the same intrinsic temperature at some epoch, we expect the subset of dereddened SNe to have nearly identical colors $\left(C_{0}\right)$; the remaining objects should show color excesses, $E(C)=C-C_{0}$, in direct proportion to their extinctions. A useful diagnostic to check our underlying assumption is the color-color plot: unreddened SNe should occupy a small region in this plane. If we further assume the same extinction law in the SN host galaxies, the subset of extinguished objects should describe a straight line originating from such a region. The figures of merit in this test are (1) the color dispersion displayed by the unreddened $\mathrm{SNe},(2)$ the slope described by the reddened $\mathrm{SNe}$ (which is determined by the extinction law), and (3) the dispersion relative to the straight line (the smaller the better).

We have identified four objects in our sample (SN 2003B, SN 2003bl, SN 2003bn, SN 2003cn) consistent with zero reddening. These objects were selected for having (1) no significant Na I D interstellar lines in their spectra at the redshifts of their host galaxies and (2) early-time spectra that show no signs of host-galaxy extinction. To judge the latter, we used extinction values determined from fits of SN IIP atmosphere models to our early-time spectra as done by Dessart \& Hillier (2006) and Dessart et al. (2008). Such models use the SN spectral lines to constrain the photospheric temperature and the continuum to restrict the amount of extinction. Examples of fits are shown in Figure 4.

We investigated two color-color plots $(V-I$ versus $V-R$, and $V-I$ versus $B-V$ ) over a wide range of epochs (from day -50 to -15$)$ after correcting the photometry for Galactic extinction and $K$-terms as described by Olivares (2008). The best results were found for the $V-I$ versus $V-R$ diagram constructed from day -30 , shown in Figure 5. Fortunately, all $\mathrm{SNe}$ in our sample have enough data to interpolate color curves at day 30. At this epoch, approximately the end of the plateau, we obtain the linear behavior expected for a sample with the same intrinsic color but different degrees of extinction. Shown with a triangle is the single $\mathrm{SN}$ consistent with zero extinction which is, remarkably, one of the bluest objects in this diagram; the other three unextinguished $\mathrm{SNe}$ do not have $R$-band photometry. A least-squares fit to the data yields a slope of $1.59 \pm 0.09$, which is lower than the $E(V-I) / E(V-R)=2.12$ ratio expected for a Galactic extinction law $\left(R_{V}=3.1\right)$ shown as a vector 


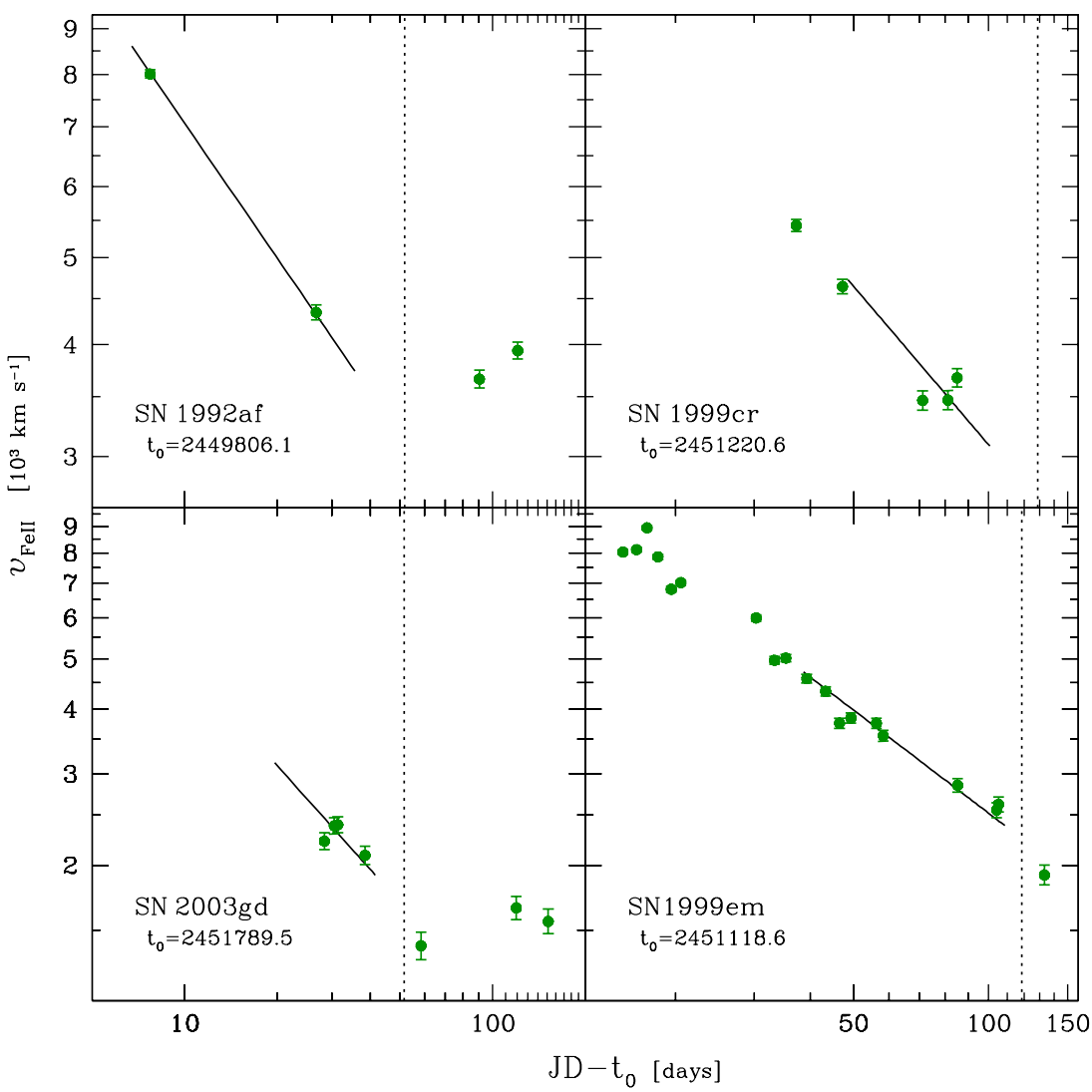

Figure 3. Four expansion velocity curves representative of the SN sample measured by means of the Fe II $\lambda 5169$ line profile. While solid lines correspond to a power-law fit (Equation (5)), dashed lines show the transition time. The $t_{0}$ parameter of the fit was chosen as the time origin due to plotting restrictions. For SN 1992af (top left panel), we fit only two velocities (see Section 3.1.3 for more details). The top right panel shows a 15 day extrapolation beyond the last data point for SN 1999cr. The bottom left panel shows a 10 day extrapolation for SN 2003gd prior to the first data point. SN 1999em velocities are shown in the bottom right panel as an example of good sampling.

(A color version of this figure is available in the online journal.)

in Figure 5. This suggests a somewhat different extinction law in the SN hosts compared to the Galaxy. The dispersion of $0.059 \mathrm{mag}$ in $V-I$ is a promising result as it translates into an uncertainty of $A_{\text {host }}(V)=0.15 \mathrm{mag}$, which corresponds to the limiting precision of this method. The reduced $\chi^{2}$ of 1.55 implies that the dispersion can be accounted almost solely by our error bars and that any intrinsic color dispersion in our sample is $\leqslant 0.06 \mathrm{mag}$. The bottom line is that both the $V-R$ and $V-I$ colors fulfill the minimum requirements as reddening indicators.

The best results from the $B-V$ versus $V-I$ analysis, obtained from day -30 , are shown in Figure 6. The four $\mathrm{SNe}$ consistent with zero extinction, plotted with triangles, have weighted average colors of $(B-V)_{0}=1.147 \pm 0.053 \mathrm{mag}$ and $(V-I)_{0}=$ $0.656 \pm 0.053 \mathrm{mag}$, where the dispersion was used as measure of the uncertainty. Note that there are five $\mathrm{SNe}$ in this diagram which are slightly bluer than the unreddened sample. The $V-I$ color dispersion of $0.076 \mathrm{mag}$ is greater than that obtained in Figure 5 and is most likely due to the $B-V$ color, since the $B$ band is more sensitive to the metallicity of the $\mathrm{SN}$, owing to many absorption lines that lie in this spectral region. Therefore, we believe that the greater dispersion in this diagram could be due to the different metallicities of our SN sample. A least-squares fit to the data yields a slope of $0.77 \pm 0.04$. This slope is quite different from the $E(V-I) / E(B-V)=1.38$ ratio expected for the Galactic extinction law (shown as a vector in Figure 6), in agreement with the departure from a standard reddening law seen in the $V-I$ versus $V-R$ diagram.
We conclude from our exploration that, while the $B-V$ color is problematic, both the $V-R$ and $V-I$ colors offer a promising route for dereddening purposes. In what follows, we will employ solely the $V-I$ color since only a small subset of our objects possess $R$-band photometry. Although the evidence points to a non-Galactic reddening law, for now we will assume a standard reddening law (later we will relax this assumption; see Section 4.3.2).

Using our library of SN II spectra, we computed synthetically the appropriate conversion factor between $E(V-I)$ and $A_{V}$ for $\mathrm{SNe}$ II and a standard reddening law $\left(R_{V}=3.1\right)$, yielding

$$
\beta_{V}=\frac{A_{V}}{E(V-I)}=2.518 .
$$

The host-galaxy extinction can be computed from $A_{V}(V-I)=$ $\beta_{V}\left[(V-I)-(V-I)_{0}\right]$ and, assuming an intrinsic color of $(V-I)_{0}=0.656 \pm 0.053 \mathrm{mag}$, its numerical expression with the corresponding uncertainty is

$$
\begin{aligned}
A_{V}(V-I) & =2.518[(V-I)-0.656], \\
\sigma\left(A_{V}\right) & =2.518 \sqrt{\sigma_{(V-I)}+0.053^{2}+0.059^{2}},
\end{aligned}
$$

where $V-I$ corresponds to the color of a given $\mathrm{SN}$ at day -30 (corrected for $K$-terms and foreground extinction) and $\sigma_{(V-I)}$ combines the instrumental errors in the $V$ and $I$ magnitudes, the uncertainty in $A_{\mathrm{G}}$ and the $K$-terms (see Olivares 2008, chapters 3.1.1 and 3.1.2, respectively), and the uncertainty in $t_{\mathrm{PT}}$ 


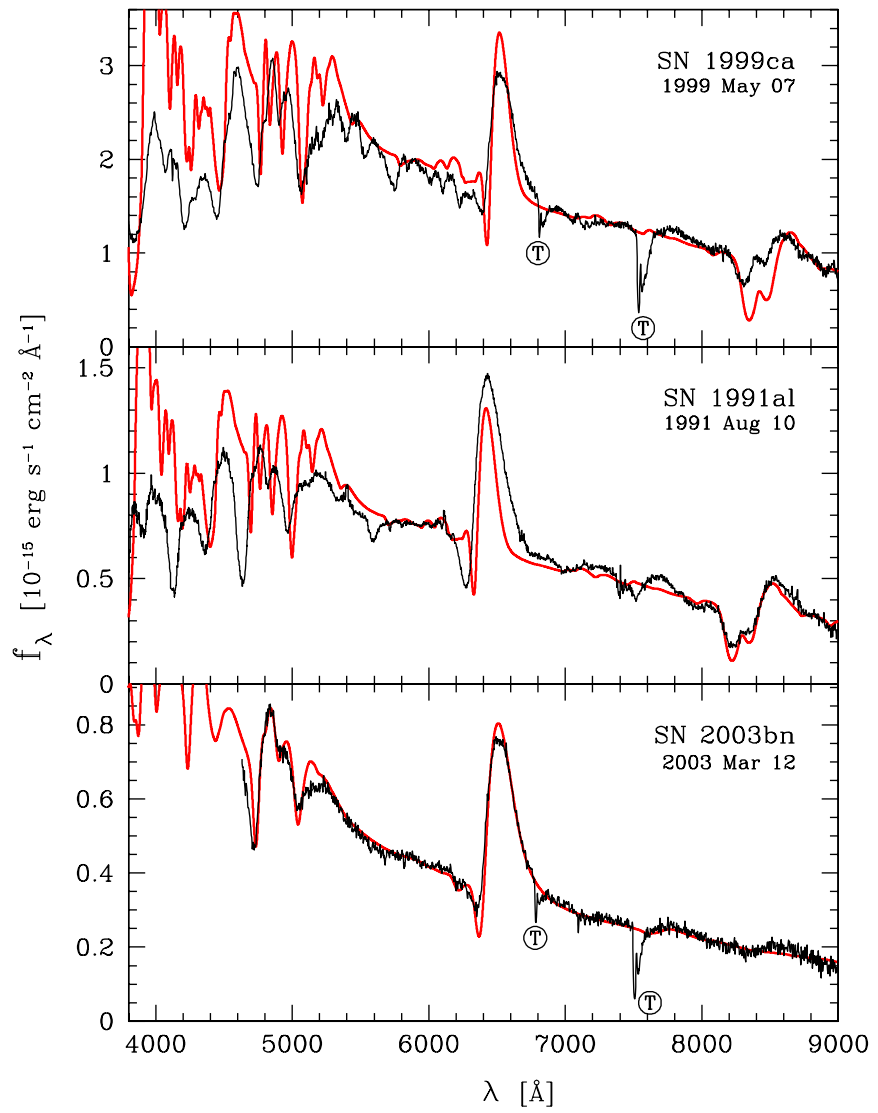

Figure 4. Type IIP SN atmosphere models (wide gray line) fitted to our spectra (black line) in the rest frame. The top panel shows an example of an unsatisfactory fit to one of our late-time spectra. The middle panel shows a better fit to an early-time spectrum of SN 1991al. A much better fit is achieved for an early-time spectrum of SN $2003 \mathrm{bl}$ as shown in the bottom panel. Strong telluric lines are marked.

(A color version of this figure is available in the online journal.)

(Section 3.1.1). The uncertainty in the intrinsic $(V-I)_{0}$ color was also included in the error of $A_{V}$ along with the $V-I$ standard deviation in the $V-I$ versus $V-R$ diagram.

The host-galaxy reddening values obtained from this technique are listed in Column 5 of Table 4 (with the uncertainties given in parentheses for the whole sample of $37 \mathrm{SNe}$ ). There are eight SNe with $V-I$ colors bluer than $(V-I)_{0}$ which stand out in this table for their inferred negative reddenings. Although not physically meaningful, these negative values are statistically consistent with zero or moderate reddenings. In fact, seven of these eight objects differ by $<1.1 \sigma$ from zero reddening. Only SN 1992af differs by $1.8 \sigma$ from $A_{\text {host }}=0$ mag.

As a comparison, Krisciunas et al. (2009) employed a similar procedure to determine the extinction to SN 2003hn. While we get $A_{V}=0.46 \pm 0.21$, they obtained $A_{V}=0.54 \pm$ 0.14 , values that are consistent within the uncertainties. In the following section, we compare this method with other dereddening techniques.

\section{ANALYSIS}

\subsection{Comparing Dereddening Techniques}

SN IIP atmosphere models were fitted to our library of spectra as done by Dessart \& Hillier (2006) and Dessart et al. (2008). In these fits the spectral lines are used to constrain the photospheric temperature, and the corresponding continuum is employed to estimate the extinction. Thus, the quality of the fit

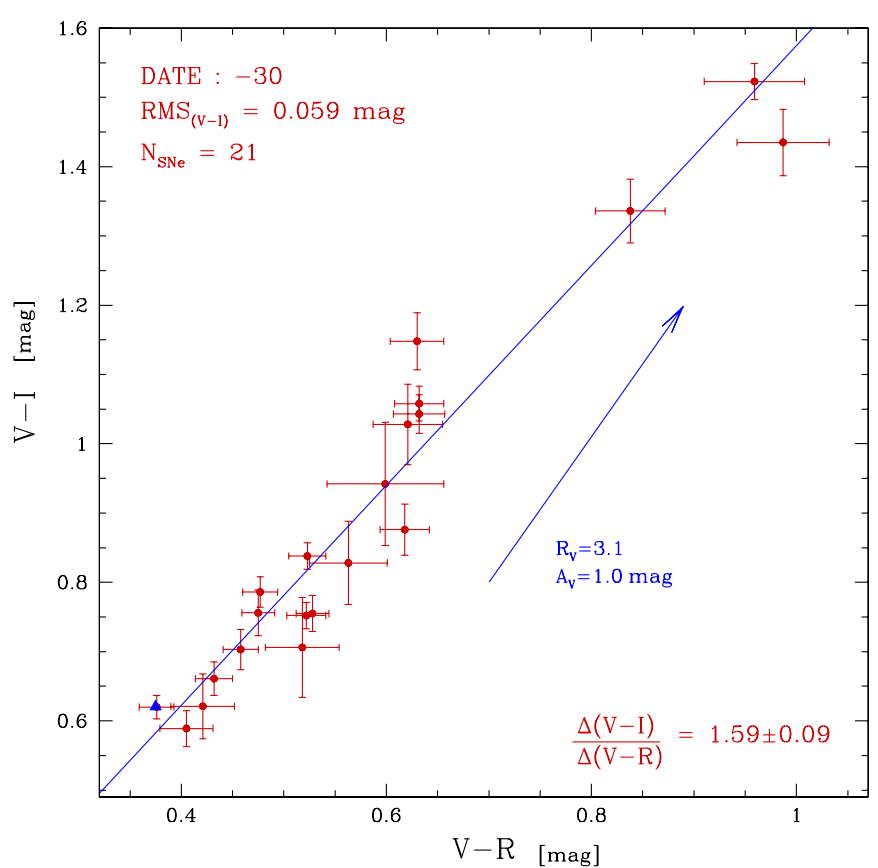

Figure 5. $V-I$ vs. $V-R$ diagram for $21 \mathrm{SNe}$ IIP having $V R I$ photometry corrected for the $A_{\mathrm{G}}$ and $K$-terms. The solid line is a least-squares fit to the data, with a slope of $1.59 \pm 0.09$. The arrow has a slope of 2.12 and corresponds to the reddening vector for $A_{V}=1.0 \mathrm{mag}$ and a standard Galactic extinction law $\left(R_{V}=3.1\right)$. The black triangle shows the one $\mathrm{SN}$ of this subsample consistent with zero extinction having $R$-band photometry.

(A color version of this figure is available in the online journal.)

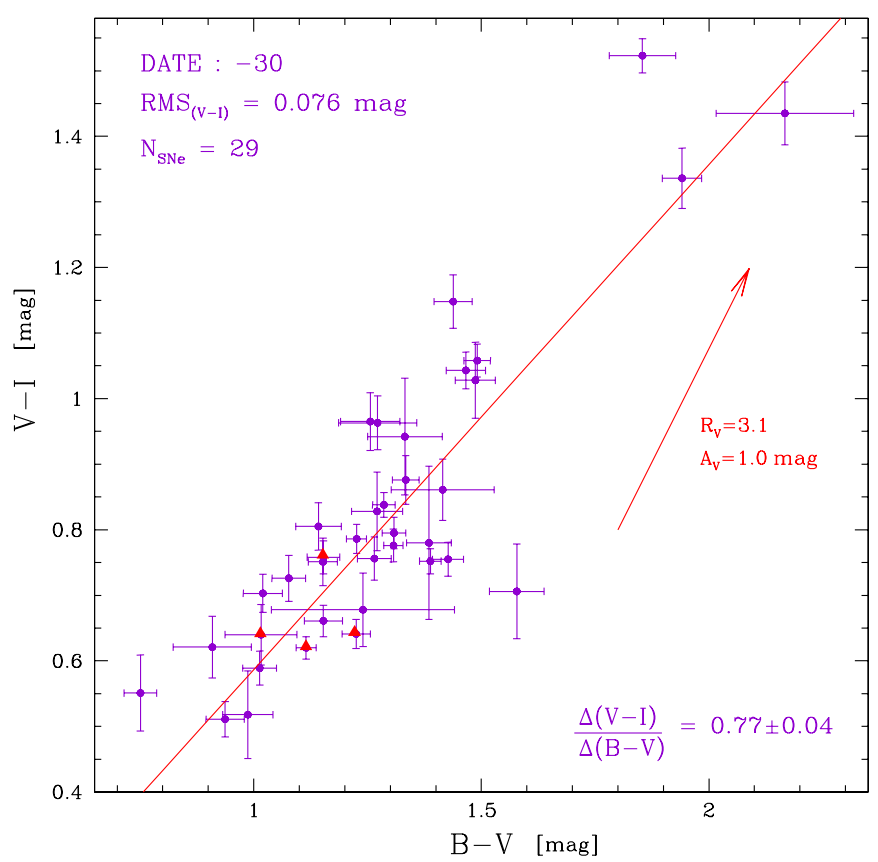

Figure 6. $V-I$ vs. $B-V$ diagram for $29 \mathrm{SNe}$ IIP having $B V I$ photometry corrected for the $A_{\mathrm{G}}$ and $K$-terms. The solid line is a least-squares fit to the data, with a slope of $0.77 \pm 0.04$. The arrow has a slope of 1.38 and corresponds to the reddening vector for $A_{V}=1.0 \mathrm{mag}$ and a standard Galactic extinction law $\left(R_{V}=3.1\right)$. The black triangles show the four $\mathrm{SNe}$ consistent with zero host-galaxy extinction.

(A color version of this figure is available in the online journal.)

depends on (1) the determination of the photospheric temperature by means of the spectral lines and (2) how well the continuum is represented by the extinguished blackbody emission. A 
Table 4

Host-galaxy Extinctions

\begin{tabular}{|c|c|c|c|c|}
\hline SN Name & $A_{V}(\text { spec })^{\mathrm{a}}$ & Subclass & $A_{V}(\mathrm{Na} \text { I D })^{\mathrm{b}}$ & $A_{V}(V-I)^{\mathrm{c}}$ \\
\hline 1991al & $0.31(16)$ & Silver & $0.31(06)$ & $-0.17(21)$ \\
\hline 1992af & $1.24(31)$ & Coal & $0.17(15)$ & $-0.37(21)$ \\
\hline $1992 \mathrm{am}$ & $\ldots$ & & $0.00(43)$ & $0.52(23)$ \\
\hline 1992ba & $0.43(16)$ & Silver & $0.00(03)$ & $0.30(21)$ \\
\hline $1993 \mathrm{~A}$ & $0.00(31)$ & Bronze & $0.00(54)$ & $0.06(25)$ \\
\hline $1999 b r$ & $0.25(16)$ & Silver & $0.00(04)$ & $0.94(25)$ \\
\hline $1999 \mathrm{ca}$ & $0.12(31)$ & Coal & $0.34(05)$ & $0.25(21)$ \\
\hline $1999 \mathrm{cr}$ & $0.47(31)$ & Coal & $0.69(21)$ & $0.12(21)$ \\
\hline $1999 \mathrm{em}$ & $0.31(16)$ & Gold & $1.01(05)$ & $0.24(21)$ \\
\hline 1999gi & $0.56(16)$ & Silver & $0.50(08)$ & $1.02(21)$ \\
\hline 0210 & $0.31(31)$ & Bronze & $0.00(23)$ & $0.31(36)$ \\
\hline $2002 \mathrm{fa}$ & $\ldots$ & & $0.00(14)$ & $-0.35(26)$ \\
\hline $2002 \mathrm{gw}$ & $0.40(19)$ & Silver & $0.00(02)$ & $0.18(22)$ \\
\hline 2002hj & $0.16(31)$ & Bronze & $0.00(06)$ & $0.24(22)$ \\
\hline $2002 \mathrm{hx}$ & $0.16(25)$ & Coal & $0.00(16)$ & $0.38(22)$ \\
\hline $2003 B^{d}$ & $0.00(25)$ & Silver & $0.12(05)$ & $-0.09(21)$ \\
\hline $2003 E$ & $1.09(31)$ & Coal & $0.71(07)$ & $0.78(23)$ \\
\hline $2003 \mathrm{~T}$ & $0.53(31)$ & Coal & $0.19(19)$ & $0.35(21)$ \\
\hline $2003 \mathrm{bl}^{\mathrm{d}}$ & $0.00(16)$ & Gold & $0.11(10)$ & $0.26(21)$ \\
\hline $2003 b^{d}$ & $0.09(16)$ & Silver & $0.00(03)$ & $-0.04(21)$ \\
\hline $2003 \mathrm{ci}$ & $0.43(31)$ & Coal & $0.00(23)$ & $0.78(23)$ \\
\hline $2003 \mathrm{cn}^{\mathrm{d}}$ & $0.00(25)$ & Gold & $0.00(09)$ & $-0.04(23)$ \\
\hline $2003 c x$ & $0.65(25)$ & Coal & $\ldots$ & $-0.27(25)$ \\
\hline 2003 ef & $1.24(25)$ & Gold & $1.40(12)$ & $0.98(21)$ \\
\hline $2003 \mathrm{fb}$ & $0.37(31)$ & Coal & $0.54(23)$ & $1.24(23)$ \\
\hline $2003 \mathrm{gd}$ & $0.40(31)$ & Coal & $0.00(04)$ & $0.33(21)$ \\
\hline 2003hd & $0.90(31)$ & Coal & $0.74(27)$ & $0.01(21)$ \\
\hline 2003hg & $\ldots$ & & $2.29(12)$ & $1.97(24)$ \\
\hline $2003 \mathrm{hk}$ & $0.65(31)$ & Coal & $1.69(20)$ & $0.44(25)$ \\
\hline $2003 \mathrm{hl}$ & $1.24(25)$ & Gold & $1.84(09)$ & $1.72(23)$ \\
\hline $2003 \mathrm{hn}$ & $0.59(25)$ & Coal & $0.64(08)$ & $0.46(21)$ \\
\hline 2003ho & $1.24(31)$ & Bronze & $1.28(10)$ & $2.19(21)$ \\
\hline 2003ip & $0.40(31)$ & Coal & $0.42(08)$ & $0.56(22)$ \\
\hline $2003 \mathrm{iq}$ & $0.37(16)$ & Silver & $0.91(04)$ & $0.25(22)$ \\
\hline $2004 d j$ & $0.50(25)$ & Coal & $0.26(06)$ & $-0.09(23)$ \\
\hline 2004 et & $0.00(25)$ & Coal & $1.17(02)$ & $0.13(27)$ \\
\hline $2005 \mathrm{cs}$ & $\ldots$ & & $\ldots$ & $0.72(30)$ \\
\hline
\end{tabular}

Notes.

a Spectroscopic reddenings assuming $R_{V}=3.1$. Additionally, the third column lists the subclass defined from the criteria given in Section 4.1.

${ }^{b} \mathrm{EW}$ of $\mathrm{Na}$ I D measured by us and converted to $A_{V}$ with the relationship of Barbon et al. (1990) using $R_{V}=3.1$.

c This paper.

${ }^{\mathrm{d}} \mathrm{SNe}$ selected to determine the intrinsic color.

crucial condition for this technique to work is the spectrophotometric quality of the spectra.

In general, our observations were obtained with the slit oriented along the parallactic angle (Filippenko 1982) and the relative shape of the spectra should be accurate. However, this was not always possible; moreover, sometimes the spectra were contaminated by light from the host galaxy. Accordingly, we first checked the flux calibration of each spectrum by synthesizing magnitudes and comparing them to the observed magnitudes, duly interpolated to the time of the spectroscopy. We typically found an agreement better than 0.05 mag between the observed and synthetic colors, thus confirming our confidence in the flux calibration. In the 64 cases ( $18 \%$ of all spectra) where we found significant differences between synthetic and observed colors $(\geqslant 0.1 \mathrm{mag}$ for any color), we applied a low-order polynomial correction to the spectrum. Basically, this "mangling" correction used the observed photometry to change the slope of a spectrum. After checking the flux calibration (and mangling it, if needed), the next step was to correct for Galactic absorption and deredshift the spectra. This database was then used for the atmosphere model fits. Examples of the fits are shown in Figure 4.

The resulting spectroscopic reddenings are summarized in Column 2 of Table 4. As pointed out by Dessart \& Hillier (2006), the spectrum-fitting technique works much better with early-time spectra than with late-time observations. At late times the photosphere has receded in mass, exposing inner and more metal-rich layers, which translates into an overabundance of heavy-element absorption lines. Therefore, the fitting to the continuum (essentially a temperature fitting) is hampered by the increased line opacity at late times. We thus divided our sample into four subcategories based on the epoch and the flux quality of the spectra used in the reddening determination: (a) gold: earlytime spectra, without mangling correction; (b) silver: early-time spectra, with mangling correction; (c) bronze: late-time spectra, without mangling correction; and (d) coal: late-time spectra, with mangling correction. Note that the main criterion is whether the spectrum was obtained at early or late times, and the second criterion corresponds to the flux-calibration quality. We consider the unmangled spectra to be of higher quality than the mangled ones, because the former do not require any corrections and suggest that the observations were better performed.

The above subclassification is given for each $\mathrm{SN}$ in Column 3 of Table 4 . Note that the uncertainty is directly related to this subclassification. Dessart et al. (2008) estimate an error of $\sigma_{E(B-V)}=0.05 \mathrm{mag}$ when using early-time spectra, and $\sigma_{E(B-V)}=0.10 \mathrm{mag}$ when using late-time spectra. We refine this argument, ramping up from 0.05 to $0.10 \mathrm{mag}$, depending on the number of spectra employed for each extinction determination. These values are given in Table 4 in units of $A_{V}=3.1 E(B-V)$.

Figure 7 shows a comparison between the spectroscopic reddenings $A_{V}$ (spec) and our color reddenings $A_{V}(V-I)$ derived in Section 3.2, for the $17 \mathrm{SNe}$ belonging to the top three subclasses (gold + silver + bronze). Good agreement is found between both techniques, with a dispersion of 0.38 mag. The resulting $\chi_{v}^{2}=1.4$ suggests that this dispersion is consistent with the combined errors of both techniques. The exceptions are two objects, SN 1999br and SN 2003ho. SN 1999br has only plateau-phase photometry, making it hard to determine $t_{\mathrm{PT}}$. Although the uncertainty in $t_{\mathrm{PT}}$ is quite large (20 days), this does not have a great impact on the error of the $V-I$ color due to the flatness of the color curve at these epochs $\left(+0.0024 \mathrm{mag} \mathrm{day}^{-1}\right)$. Another possible reason for the disagreement could be an intrinsically redder $V-I$ color than that of the bulk of the SNe, caused by the extreme properties (low luminosity and expansion velocity) of SN 1999br. The second discrepant object (SN 2003ho) belongs to the bronze group, so it is possible that the difference could be due to the use of a late-time spectrum in the determination of the spectroscopic reddening.

Another way to estimate host-galaxy reddening is from the Na I D $\lambda \lambda 5889,5896$ interstellar absorption doublet observed in the SN spectrum at the host-galaxy redshift. Whenever the line was detected we measured its equivalent width (EW); in those cases where we did not detect the Na I D line we assigned it a null value. In all cases, we estimated the uncertainty in the EW based on the $\mathrm{S} / \mathrm{N}$ of the continuum around this line. We converted these measurements into visual extinctions, $A_{V}(\mathrm{Na}$ I D), using the calibration of Barbon et al. (1990):

$$
E(B-V) \approx 0.25 \mathrm{EW}(\mathrm{Na} \text { I D }),
$$

where the units of $E(B-V)$ and EW are mag and $\AA$, re- 


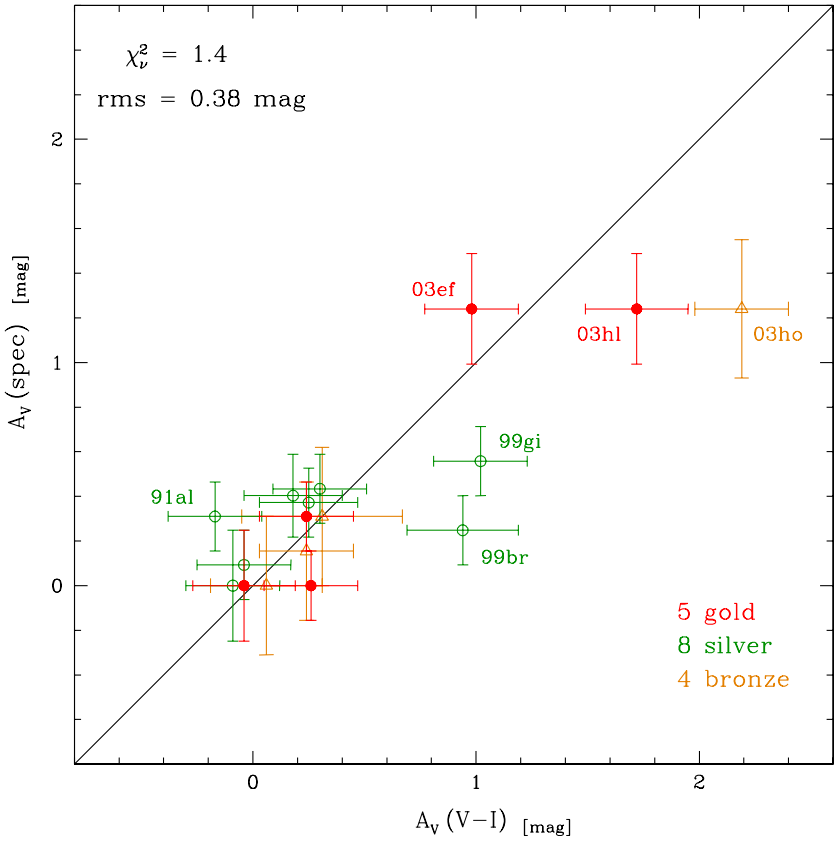

Figure 7. Comparison between two dereddening techniques via $A_{V}$ (mag): the spectrum-fitting method (ordinate) and our technique (abscissa; Section 3.2). Shown are three subclasses defined by the quality of the spectroscopic data used: the gold (gray filled circles), silver (light gray open circles), and bronze (black triangles) samples (see Section 4.1). The solid line is fixed to show a slope of unity.

(A color version of this figure is available in the online journal.)

spectively. Although Turatto et al. (2003) showed that this relation is bimodal, and Blondin et al. (2009) found that the $E(B-V)$ estimates based on $\mathrm{EW}(\mathrm{Na}$ I $\mathrm{D})$ were highly inaccurate, the $E(B-V)$ values derived from Equation 8 (tabulated in Column 4 of Table 4) provide a rough estimate of $E(B-V)$ to compare with our results. The comparison between $A_{V}(\mathrm{Na}$ I D $)$ and our technique, shown in Figure 8, exhibits a dispersion of $0.53 \mathrm{mag}\left(\chi_{v}^{2}=3.8\right)$, which is much higher than the $\sigma=$ $0.38 \mathrm{mag}$ obtained from the previous comparison. The disagreement can be attributed to the fact that the absorption line traces the gas content along the line of sight, but does not necessarily probe dust (Munari \& Zwitter 1997). Furthermore, reddenings derived from the $\mathrm{EW}$ of the Na I D lines measured from our lowdispersion spectra simply cannot be expected to be accurate; the D lines produced by a typical interstellar cloud are saturated (Hobbs 1974). The only way that one can hope to derive a reliable reddening from the D lines is via very high-dispersion spectra that resolve the lines and allow the column density to be determined.

\subsection{Luminosity versus Expansion Velocity}

After developing the dereddening method based on latetime colors, we can now revisit the LEV relation originally discovered by Hamuy \& Pinto (2002), which is at the core of the SCM. For this purpose, we applied $A K A$ corrections to our photometry (Olivares 2008, Chapter 3.1), we used our analytic fits (Section 3.1.1) to interpolate BVI magnitudes on day -30 , and we employed the cosmic microwave background (CMB) redshifts ${ }^{10}$ in Table 5 to convert apparent magnitudes to absolute

\footnotetext{
10 The CMB redshifts were computed by adding the heliocentric redshifts given in Table 2 and the projection of the velocity of the Sun relative to the $\mathrm{CMB}$ in the direction of the SN host galaxy. For the latter, we adopted a velocity of $371 \mathrm{~km} \mathrm{~s}^{-1}$ in the direction $(l, b)=(264.1,48.3)$ given by Fixsen et al. (1996) plus the uncertainty of the Local Group velocity $\left(187 \mathrm{~km} \mathrm{~s}^{-1}\right)$.
}

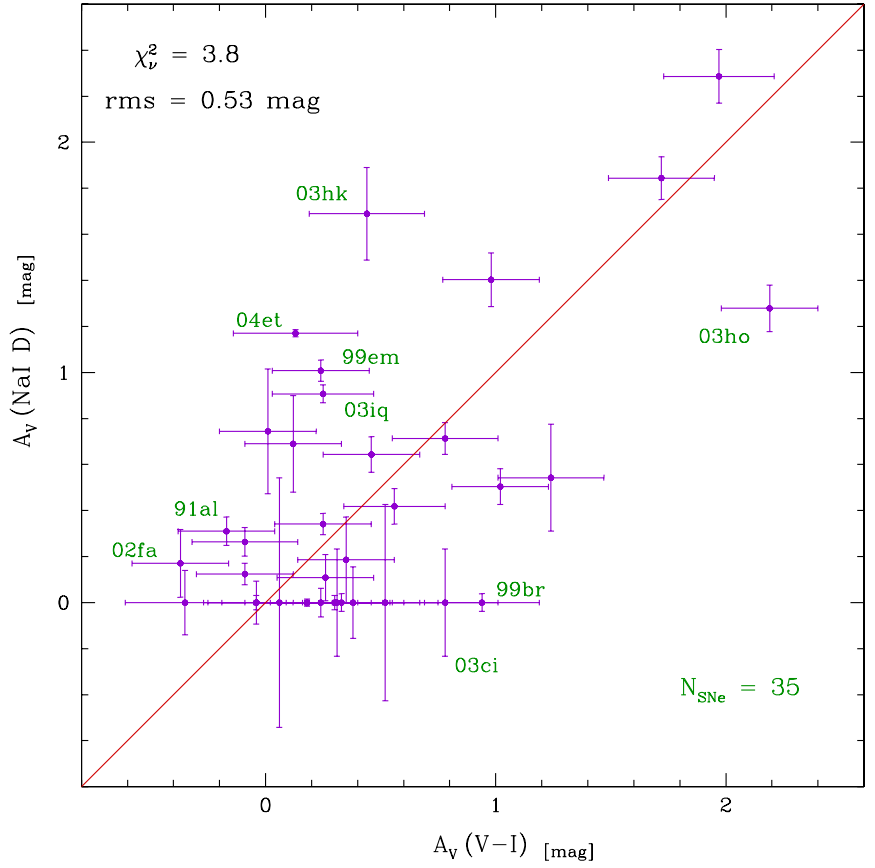

Figure 8. Comparison between two dereddening techniques via $A_{V}$ (mag): the $\mathrm{Na}$ I D interstellar line (ordinate) and our technique (abscissa; Section 3.2). In each case, the EW of the Na I interstellar line is transformed into a visual extinction according to the calibration given by Barbon et al. (1990).

(A color version of this figure is available in the online journal.)

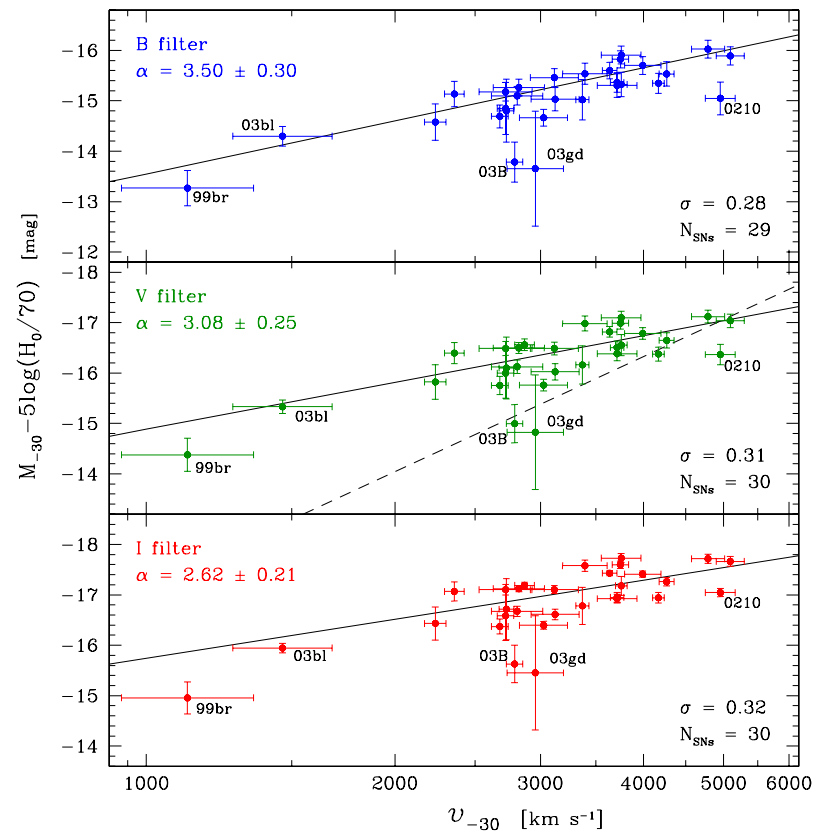

Figure 9. $B V I$-band absolute magnitudes (ordinate) against the Fe II expansion velocity (abscissa) for 29-30 SNe. We use magnitudes corrected for Galactic extinction, $K$-terms, and host-galaxy extinction together with a value of the Hubble constant of $70 \mathrm{~km} \mathrm{~s}^{-1} \mathrm{Mpc}^{-1}$. The dashed line in the middle panel represents the LEV relation for the $V$ band found by Hamuy \& Pinto (2002), obtained from magnitudes and velocities measured at day 50 past the explosion (approximately day -60 on our own timescale).

(A color version of this figure is available in the online journal.)

values (assuming $H_{0}=70 \mathrm{~km} \mathrm{~s}^{-1} \mathrm{Mpc}^{-1}$ from our final result). In order to assess the dereddening technique, we preserved the relative reddening estimations by keeping the negative values of host-galaxy absorption. We performed a power-law fit to interpolate a velocity contemporaneous with the photometry 
(day -30) as described in Section 3.1.1. Only for SN 1999cr did we have to use an extrapolated velocity (see top right panel in Figure 3). From our original sample of $37 \mathrm{SNe}$ IIP we were able to use $30 \mathrm{SNe}$ to build this relation, since five of them do not have measured $\mathrm{Fe}$ II velocities at day -30 , and two others have extremely low redshifts $\left(c z_{\mathrm{CMB}}<300 \mathrm{~km} \mathrm{~s}^{-1}\right)$.

Figure 9 shows the resulting LEV relation (absolute magnitude versus expansion velocity) for all $B V I$ bands. Evidently we recover the result of Hamuy \& Pinto (2002), namely that the most luminous $\mathrm{SNe}$ have greater expansion velocities. In their case the data were modeled with a linear function. Our sample suggests that the relation may be quadratic, but we need more $\mathrm{SNe}$ at low expansion velocities to confirm this suspicion. Linear least-squares fits to our $B V I$ data yield the following solutions:

$$
\begin{aligned}
& M(B)_{-30}-5 \log \left(H_{0} / 70\right)=3.50( \pm 0.30) \\
& \quad \times \log \left[v_{\text {FeII }}(-30) / 5000\right]-16.01( \pm 0.20), \\
& M(V)_{-30}-5 \log \left(H_{0} / 70\right)=3.08( \pm 0.25) \\
& \quad \times \log \left[v_{\text {FeII }}(-30) / 5000\right]-17.06( \pm 0.14), \\
& M(I)_{-30}-5 \log \left(H_{0} / 70\right)=2.62( \pm 0.21) \\
& \quad \times \log \left[v_{\text {FeII }}(-30) / 5000\right]-17.61( \pm 0.10),
\end{aligned}
$$

which are shown with solid lines in Figure 9. The relation found by Hamuy \& Pinto (2002) for the $V$ band is indicated with the dashed line in the middle panel of the same figure. Given that the study of Hamuy \& Pinto (2002) was performed using data at day 50 after explosion (around day -60 on our time scale), it is not unexpected that their LEV relation is shifted to higher expansion velocities. Some of the difference in slope is explained by the inclusion of SN 2003bl in our sample, which flattens the correlation. This relation exhibits a dispersion of $0.3 \mathrm{mag}$, similar to that reported before. Such low scatter is very encouraging as it implies that the expansion velocities can be used to predict the SN luminosities, to standardize them, and to derive distances.

Recently, Kasen \& Woosley (2009) have computed hydrodynamical models for SNe IIP, which support the LEV relation initially reported by Hamuy \& Pinto (2002). Also, M. Bersten (2010, in preparation) has used her own hydrodynamical models to investigate the LEV relationship. By varying the explosion energy, she has obtained an LEV relation with a slope consistent with that reported here.

\subsection{Hubble Diagrams}

In order to assess the performance of the SCM, we employ (1) host-galaxy redshifts in the CMB frame with an uncertainty of $187 \mathrm{~km} \mathrm{~s}^{-1}$, (2) $B V I$ apparent magnitudes and $V-I$ colors corrected for $K$-terms and Galactic reddening, and (3) Fe II velocities (in units of $\mathrm{km} \mathrm{s}^{-1}$ ). Table 5 lists these values for the $37 \mathrm{SNe}$ of our sample, of which 35 meet an initial requirement of $c z_{\mathrm{CMB}}>300 \mathrm{~km} \mathrm{~s}^{-1}$. The weighted dispersion of the data points around a linear fit in the HDs allows us to evaluate the precision of different approaches.

$$
\text { 4.3.1. Using } A_{V}(V-I) \text { and } A_{V}(\text { spec })
$$

The top left panel of Figure 10 shows an HD constructed from $B$-band magnitudes interpolated to day -30 . The top right panel shows magnitudes additionally corrected for the LEV relation, and the bottom left panel includes further corrections for host-galaxy extinction (using the $V-I$ color calibration given in Section 3.2). In each case, we perform a weighted linear leastsquares fit of the form

$$
m+\alpha \log \left(v_{\mathrm{FeII}} / 5000\right)-A_{\text {host }}=5 \log c z+z p,
$$

where $m$ is the apparent magnitude corrected for $K$-terms and Galactic absorption, $v_{\text {FeII }}$ is the expansion velocity, $A_{\text {host }}$ is the host-galaxy absorption, and $z$ is the CMB redshift. Note that the $\mathrm{SN}$ redshift enters logarithmically in this model, implying that low-redshift SNe carry less weight in the fit. The only fitting parameters are $\alpha$ and the zero point ( $z p$ ), but in the top left panel we set $\alpha=0$ and we fit only for the $z p$. The significant decrease in the dispersion, from 0.72 to $0.36 \mathrm{mag}$, clearly demonstrates the need to add the velocity and host-galaxy extinction terms.

An inspection of the $V$-band diagram (Figure 11) shows a large scatter of $0.54 \mathrm{mag}$ in the top left panel. When we correct for expansion velocities, the scatter drops to $0.50 \mathrm{mag}$. This is certainly not unexpected given the LEV relation reported in the previous section. It is encouraging to note that the dispersion drops from 0.50 to $0.45 \mathrm{mag}$ when we include our host-galaxy extinction corrections; this indicates the utility of our colorbased dereddening technique. The reduced $\chi^{2}$ value of 2.45 implies that most of the scatter is caused by the observational errors. We performed the same analysis using other epochs and found that day -30 yielded the lowest dispersion.

If we turn our attention to the $I$ band (Figure 12), the final dispersion is $0.45 \mathrm{mag}$, identical to that found in the $V$ band. It seems that the dispersion could have some dependence on wavelength, since it decreases from 0.45 in $V I$ to $0.36 \mathrm{mag}$ in $B$. The scatter of $\sim 0.4 \mathrm{mag}$ in $B V I$ is comparable to, but somewhat larger than, the $\sim 0.34$ mag dispersion obtained in previous SCM studies (Hamuy \& Pinto 2002; Hamuy 2003b, 2005). It is important to note that the dispersions are independent of the intrinsic $(V-I)_{0}$ color calculated in Section 3.2.

In the bottom right panels of Figures 10-12 we examine the SCM using the spectroscopic reddenings determined for a set of $28 \mathrm{SNe}$ IIP from Table 4 instead of our color-based extinctions. The resulting dispersions in $B V I$ are $(0.67,0.54$, $0.42 \mathrm{mag}$ ) compared with $(0.36,0.45,0.45 \mathrm{mag})$ when using color-based extinctions. All of the fitting parameters derived from both dereddening techniques are compiled in Table 6 .

\subsubsection{Leaving $R_{V}$ as a Free Parameter}

The previous analysis suggests that the dispersions are somewhat larger than the observational errors. One possible source of scatter could be the reddening law. As shown in Section 3.2 and Figure 5, we have evidence for a somewhat different extinction law in the SN hosts compared to the Galaxy. Here, we take this idea a step further and analyze the HD leaving $R_{V}$ as a free parameter. To accomplish this we model the data with the expression

$$
m+\alpha \log \left(v_{\mathrm{FeII}} / 5000\right)-\beta(V-I)=5 \log c z+z p .
$$

Note that we are replacing $A_{\text {host }}$ in Equation (12) with the term $\beta(V-I)$. The $\beta$ factor is a new free parameter to be marginalized along with $\alpha$ and $z p$, and $V-I$ is the color on day -30 uncorrected for host-galaxy dust absorption. This is the same approach adopted by Tripp (1998) for SNe Ia but using decline rate instead of expansion photospheric velocity. The underlying assumption in our case is that the absolute magnitudes of SNe IIP are a two-family function of Fe II expansion velocity and $V-I$ color. The latter is interpreted as a 
Table 5

Magnitudes, Expansion Velocities, and $V-I$ Colors for Day -30

\begin{tabular}{|c|c|c|c|c|c|c|}
\hline SN Name & $\begin{array}{c}c z_{\mathrm{CMB}}{ }^{\mathrm{a}} \\
\pm 187\left(\mathrm{~km} \mathrm{~s}^{-1}\right)\end{array}$ & $\begin{array}{c}B \\
(\mathrm{mag}) \\
\end{array}$ & $\begin{array}{c}V \\
(\mathrm{mag})\end{array}$ & $\begin{array}{c}I \\
(\mathrm{mag})\end{array}$ & $\begin{array}{c}v_{\text {FeII }} \\
\left(\mathrm{km} \mathrm{s}^{-1}\right)\end{array}$ & $\begin{array}{c}V-I \\
(\mathrm{mag})\end{array}$ \\
\hline 1991al & 4480 & $17.944(036)$ & $16.917(029)$ & $16.333(020)$ & $5328(202)$ & $0.589(022)$ \\
\hline 1992af & 5359 & $18.024(038)$ & $17.096(029)$ & $16.706(053)$ & $4529(212)$ & $0.511(027)$ \\
\hline 1992am & 14007 & $20.443(117)$ & $19.053(038)$ & $18.218(033)$ & $\ldots$ & $0.830(046)$ \\
\hline $1992 \mathrm{ba}$ & 1245 & $16.962(040)$ & $15.657(031)$ & $14.886(022)$ & $2237(068)$ & $0.776(021)$ \\
\hline $1993 \mathrm{~A}$ & 8907 & $20.636(127)$ & $19.309(089)$ & $18.657(057)$ & $\ldots$ & $0.678(047)$ \\
\hline $1999 \mathrm{br}$ & 1285 & $19.010(022)$ & $17.582(013)$ & $16.574(008)$ & $1127(205)$ & $1.028(011)$ \\
\hline $1999 \mathrm{ca}$ & 3108 & $17.901(071)$ & $16.489(055)$ & $15.735(034)$ & $\ldots$ & $0.755(025)$ \\
\hline $1999 \mathrm{cr}$ & 6363 & $19.435(067)$ & $18.418(051)$ & $17.723(035)$ & $3095(207)$ & $0.703(025)$ \\
\hline $1999 \mathrm{em}$ & 670 & $15.331(034)$ & $13.998(023)$ & $13.245(017)$ & $2727(056)$ & $0.752(019)$ \\
\hline $1999 \mathrm{gi}$ & 831 & $16.554(032)$ & $15.060(019)$ & $14.011(022)$ & $2725(061)$ & $1.058(025)$ \\
\hline 0210 & 15082 & $21.955(044)$ & $20.572(028)$ & $19.733(035)$ & 4923(206) & $0.780(110)$ \\
\hline $2002 \mathrm{fa}$ & 17847 & $21.222(072)$ & $20.243(052)$ & $19.709(088)$ & $4176(061)$ & $0.518(065)$ \\
\hline $2002 \mathrm{gw}$ & 2878 & $18.575(020)$ & $17.491(029)$ & $16.752(014)$ & $2669(063)$ & $0.726(035)$ \\
\hline $2002 \mathrm{hj}$ & 6869 & $19.705(084)$ & $18.582(061)$ & $17.939(042)$ & $3657(065)$ & $0.751(031)$ \\
\hline $2002 h x$ & 9573 & $20.328(056)$ & $19.164(035)$ & $18.360(028)$ & $3960(203)$ & $0.805(031)$ \\
\hline 2003B & 1105 & $17.078(022)$ & $15.966(018)$ & $15.341(015)$ & 2795(067) & $0.620(017)$ \\
\hline $2003 E$ & 4380 & $19.659(046)$ & $18.368(026)$ & $17.472(024)$ & $2869(203)$ & $0.965(024)$ \\
\hline $2003 \mathrm{~T}$ & 8662 & $20.541(028)$ & $19.227(020)$ & $18.423(022)$ & 2803(203) & $0.795(024)$ \\
\hline $2003 \mathrm{bl}$ & 4652 & $20.158(042)$ & $18.961(020)$ & $18.230(019)$ & $1475(202)$ & $0.758(025)$ \\
\hline $2003 b n$ & 4173 & $18.638(045)$ & $17.403(034)$ & $16.769(024)$ & $2719(202)$ & $0.641(020)$ \\
\hline $2003 \mathrm{ci}$ & 9468 & $\ldots$ & $19.634(035)$ & $18.648(029)$ & $2876(077)$ & $0.963(034)$ \\
\hline $2003 \mathrm{cn}$ & 5753 & $19.852(024)$ & $18.827(015)$ & $18.182(014)$ & $2510(207)$ & $0.640(017)$ \\
\hline $2003 c x$ & 11282 & $20.417(100)$ & $19.513(050)$ & $19.050(067)$ & $3734(205)$ & $0.551(058)$ \\
\hline $2003 \mathrm{ef}$ & 4504 & $19.304(032)$ & $17.837(024)$ & $16.792(017)$ & $\ldots$ & $1.043(015)$ \\
\hline $2003 \mathrm{fb}$ & 4996 & $20.521(122)$ & $19.085(092)$ & $17.940(056)$ & $3120(206)$ & $1.148(040)$ \\
\hline $2003 \mathrm{gd}$ & 359 & $15.208(045)$ & $13.965(036)$ & $13.167(023)$ & $2976(230)$ & $0.786(019)$ \\
\hline 2003hd & 11595 & $20.462(031)$ & $19.320(014)$ & $18.658(018)$ & $3741(069)$ & $0.661(022)$ \\
\hline 2003hg & 3921 & $20.247(091)$ & $18.067(040)$ & $16.603(025)$ & $3398(211)$ & $1.435(027)$ \\
\hline 2003hk & 6568 & $19.478(036)$ & $18.201(024)$ & $17.380(023)$ & $3618(087)$ & $0.828(026)$ \\
\hline $2003 \mathrm{hl}$ & 2198 & $19.124(062)$ & $17.219(038)$ & $15.806(027)$ & $2354(063)$ & $1.336(027)$ \\
\hline $2003 \mathrm{hn}$ & 1102 & $16.416(020)$ & $15.153(011)$ & $14.307(015)$ & $3121(074)$ & $0.836(018)$ \\
\hline 2003ho & 4134 & $20.779(084)$ & $18.946(026)$ & $17.419(016)$ & $4152(080)$ & $1.523(022)$ \\
\hline 2003ip & 5050 & $18.894(046)$ & $17.549(035)$ & $16.664(025)$ & $3813(204)$ & $0.876(021)$ \\
\hline $2003 i q$ & 2198 & $17.396(049)$ & $16.124(037)$ & $15.362(024)$ & $3482(063)$ & $0.756(018)$ \\
\hline 2004dj & 180 & $12.973(074)$ & $12.046(035)$ & $11.416(033)$ & $2725(202)$ & $0.621(043)$ \\
\hline $2004 \mathrm{et}$ & -133 & $13.683(220)$ & $12.099(171)$ & $11.378(104)$ & $2901(202)$ & $0.707(070)$ \\
\hline $2005 \mathrm{cs}$ & 635 & $16.099(062)$ & $14.749(051)$ & $13.824(055)$ & $\ldots$ & $0.957(067)$ \\
\hline
\end{tabular}

Notes. All of the data in this table have been interpolated to day -30 with errors accounting for the interpolation, the error in $t_{\mathrm{PT}}$, and the instrumental error. $B V I$ corresponds to apparent magnitudes corrected for Galactic absorption and $K$-terms, but uncorrected for host-galaxy extinction. The $V-I$ colors do not exactly match the $V$ minus $I$ magnitude difference, since light and color curves are interpolated independently.

${ }^{a}$ The Velocity Calculator tool at the NASA/IPAC Extragalactic Database Web page computes the CMB redshift ( $c z_{\mathrm{CMB}}$ in $\mathrm{km} \mathrm{s}^{-1}$ ) given the coordinates and the heliocentric redshift of an object. Its error corresponds to the uncertainty in the determination of the Local Group velocity $\left(187 \mathrm{~km} \mathrm{~s}^{-1}\right)$.

color excess which varies according to dust in the host galaxy. Once $\beta$ is known we can use it to solve for host-galaxy extinction from $A(\lambda)=\beta[(V-I)-0.656]$, where 0.656 is the intrinsic $V-I$ color of SNe IIP (see Equations (6) and (7) in Section 3.2).

Figure 13 shows the $B V I$ HDs for our set of $30 \mathrm{SNe}$ IIP. We obtain dispersions of $(0.28,0.31,0.32 \mathrm{mag})$ in $B V I$, respectively, compared with $(0.36,0.45,0.45 \mathrm{mag})$ when $R_{V}$ is kept fixed. The $\chi_{v}^{2}$ values increase from $(1.6,2.5,2.5)$ to $(2.8,5.4,8.7)$. This change is due to the fact that we are not using the intrinsic color, which gives the major contribution to the errors, even more in the $I$ band. Although we expect a reduction in the scatter due to the inclusion of an additional parameter, the large drop in the dispersion is remarkable. The last three lines of Table 6 show the parameters we obtain by minimizing the dispersion in the HD for each band. When we restrict the sample to objects with $c z_{\mathrm{CMB}}>3000 \mathrm{~km} \mathrm{~s}^{-1}$ (leaving aside SNe with potentially greater peculiar velocities), we end up with $19 \mathrm{SNe}$ in the $B$ band and $20 \mathrm{SNe}$ in the $V I$ bands. The resulting HDs in $B V I$ show dispersions of $(0.25,0.28,0.30 \mathrm{mag})$, respectively, and the zero points of the HDs change by only $0.02 \mathrm{mag}$. Not surprisingly, these are lower than the $(0.28,0.31,0.32 \mathrm{mag})$ dispersions obtained from the whole sample.

Efforts to remove the peculiar velocities of low-redshift galaxies were made by means of the parametric model for peculiar flows of Tonry et al. (2000). The ingredients of this model are (1) the observed recession velocity of the galaxy, (2) the Hubble constant, and (3) the surface-brightness-fluctuations distance from Tonry et al. (2001). The output is an estimate of the peculiar velocity of the galaxy (see Hamuy 2003a, Section 1.4 for details). After correcting 10 host galaxies in our sample, the results are not conclusive. The scatter in the HDs decreased only 0.01 mag for the $I$ band.

Our assumption is that the term $\beta(V-I)$ in Equation (13) corresponds to the extinction in a broadband magnitude (with 


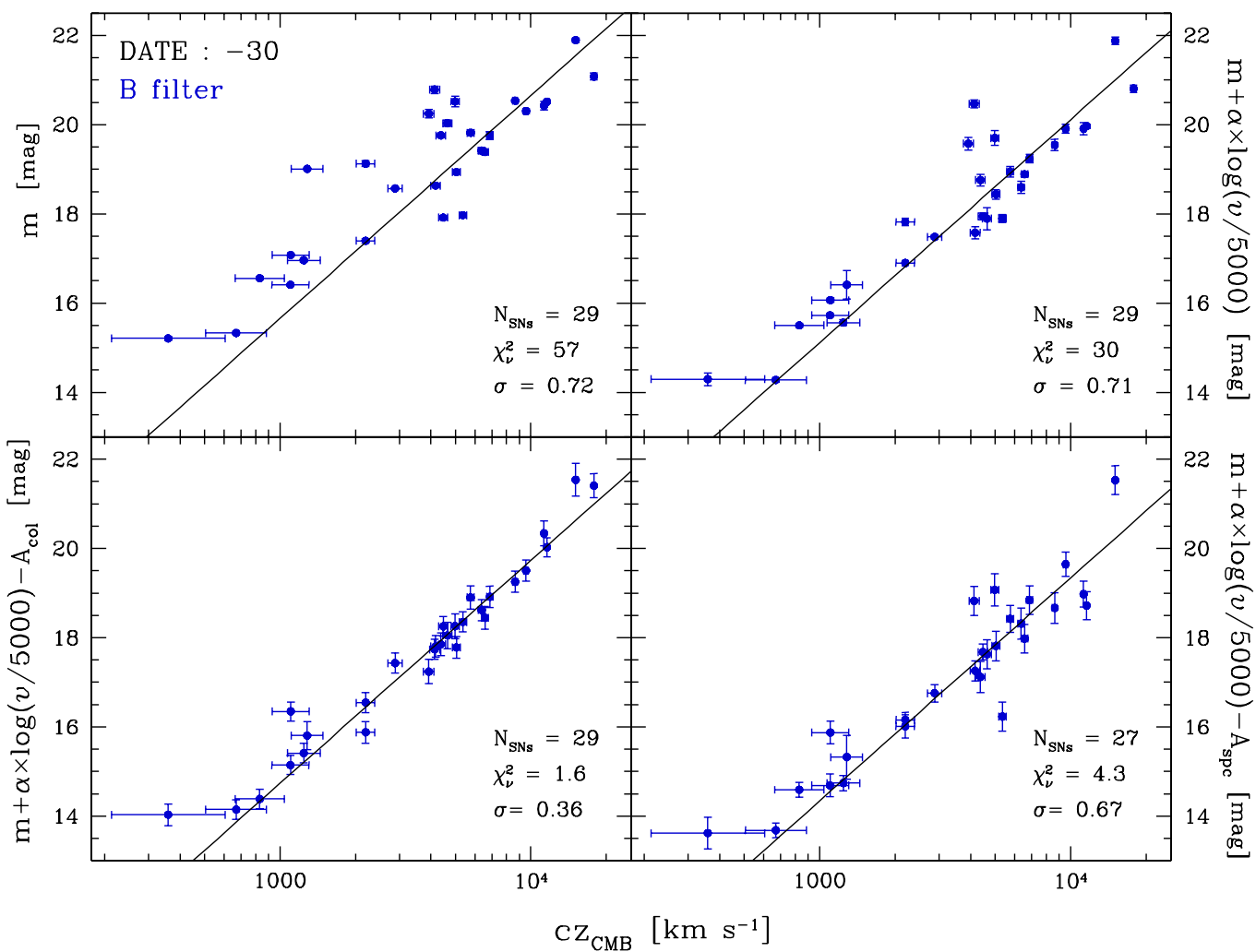

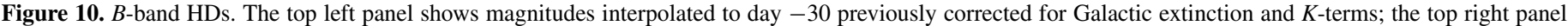

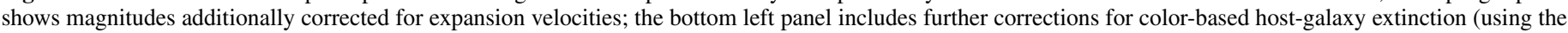

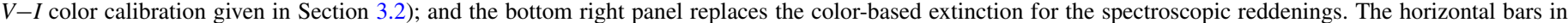
each panel correspond to an adopted uncertainty of $187 \mathrm{~km} \mathrm{~s}^{-1}$ in the CMB redshift.

(A color version of this figure is available in the online journal.)

central wavelength $\lambda$ ). Thus, $\beta$ is the ratio $A(\lambda) / E(V-I)$ (see Equation (6) in Section 3.2) and is related to the shape of the extinction law. For each of the BVI bands, we used our library of SN II spectra to compute synthetically the value of $\beta_{\lambda}$ as a function of $R_{V}=A_{V} / E(B-V)$; see Figure 14. This allowed us to convert the $\beta_{\lambda}$ values resulting from our fits into $R_{V}$ values. Our fits yield $\beta_{\lambda}=(2.67 \pm 0.13,1.67 \pm 0.10,0.60 \pm 0.09)$ for the $B V I$ bands, respectively, which translate into $R_{V}=$ $\left(1.38_{-0.24}^{+0.27}, 1.44_{-0.14}^{+0.13}, 1.36_{-0.12}^{+0.11}\right)$. These values are remarkably consistent with each other and significantly lower than the $R_{V}=3.1$ value of the standard reddening law. Independent evidence for a low- $R_{V}$ law has already been reported from studies of SNe Ia (see Section 5).

The dispersion of $0.28-0.32 \mathrm{mag}$ in the HDs translates into a precision of $13 \%-14 \%$ in the determination of relative distances. However, as noticed after inspection of Figure 15, the magnitude residuals are highly correlated among $B V I$ and generally decrease with increasing redshift. The likely explanation is that we are seeing the effects of peculiar velocities of the host galaxies, in which case the precision of the SCM could be better than the $13 \%-14 \%$ derived from the scatter in the HDs. To examine this point, for each SN we took the $c z_{\mathrm{CMB}}$ values from Table 5, and calculated the distance modulus residual

$$
\Delta \mu=m+\alpha \log \left(v_{\text {FeII }} / 5000\right)-\beta(V-I)-5 \log c z-z p,
$$

for all $B V I$ bands. Figure 16 shows strong correlations of these magnitude differences against each other. The rms scatter in the $\Delta \mu(B)$ versus $\Delta \mu(V)$ relation is $0.12 \mathrm{mag}$, and for $\Delta \mu(B)$ versus $\Delta \mu(I)$ it is $0.19 \mathrm{mag}$. This translates into $6 \%$ and $9 \%$
Table 6

Fitting Parameters from the HDs

\begin{tabular}{lcccc}
\hline \hline $\begin{array}{c}\text { Reddening } \\
\text { Estimator }\end{array}$ & Filter & $\alpha$ & $\beta$ & $z p$ \\
\hline$(V-I)^{\mathrm{a}}$ & $B$ & $3.27 \pm 0.37$ & $\ldots$ & $-0.24 \pm 0.08$ \\
& $V$ & $3.01 \pm 0.36$ & $\ldots$ & $-1.36 \pm 0.08$ \\
& $I$ & $2.99 \pm 0.36$ & $\ldots$ & $-2.02 \pm 0.08$ \\
\hline Spectral & $B$ & $4.77 \pm 0.47$ & $\ldots$ & $-0.60 \pm 0.11$ \\
analysis ${ }^{\mathrm{b}}$ & $V$ & $4.22 \pm 0.45$ & $\ldots$ & $-1.60 \pm 0.10$ \\
& $I$ & $3.67 \pm 0.43$ & $\ldots$ & $-2.11 \pm 0.10$ \\
\hline$(V-I)+$ & $B$ & $3.50 \pm 0.30$ & $2.67 \pm 0.13$ & $-1.99 \pm 0.11$ \\
variable $R_{V}{ }^{\mathrm{c}}$ & $V$ & $3.08 \pm 0.25$ & $1.67 \pm 0.10$ & $-2.38 \pm 0.09$ \\
& $I$ & $2.62 \pm 0.21$ & $0.60 \pm 0.09$ & $-2.23 \pm 0.07$
\end{tabular}

Notes. These are the results of fitting Equations (12) and (13) to the data in Tables 4 and 5.

a Reddenings from Section 3.2.

b Spectroscopic reddenings.

${ }^{\mathrm{c}}$ Leaving $R_{V}$ as a free parameter.

in distance, respectively. If this correlation of the residuals is due to peculiar velocities, then the true distance precision of $\mathrm{SCM}$ is $6 \%-9 \%$, and not $13 \%-14 \%$ as derived from the HDs (which include scatter produced by the peculiar velocities). This result is very close to the $\sim 7 \%-10 \%$ precision of methods using SNe Ia (e.g., Phillips 1993; Hamuy et al. 1996; Riess et al. 1996; Phillips et al. 1999) when they were first used to reveal the acceleration of the universe, which demonstrates that $\mathrm{SNe}$ IIP have great potential for determining extragalactic distances and therefore cosmological parameters. 


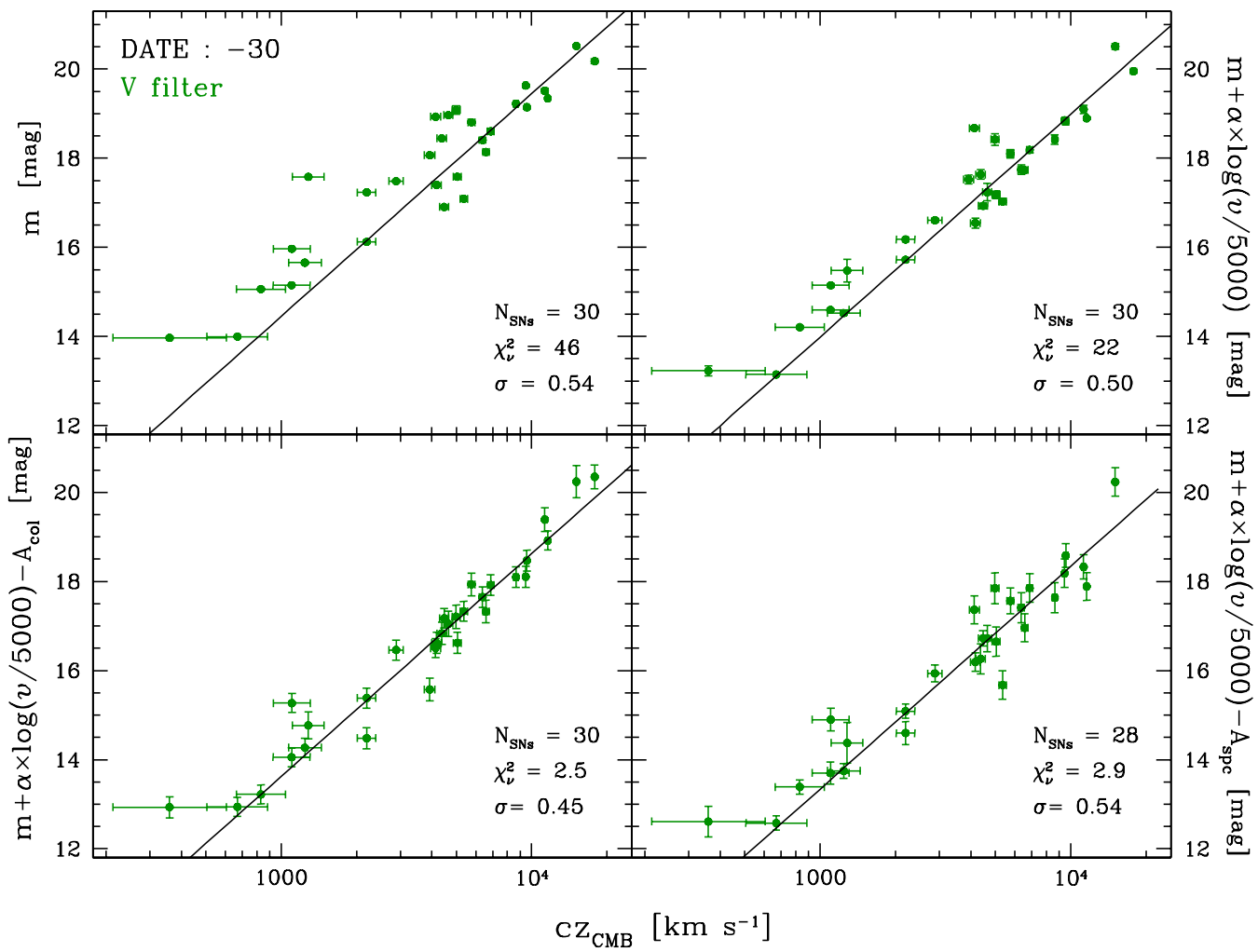

Figure 11. $V$-band HDs. The top left panel shows magnitudes interpolated to day -30 previously corrected for Galactic extinction and $K$-terms; the top right panel shows magnitudes additionally corrected for expansion velocities; the bottom left panel includes further corrections for color-based host-galaxy extinction (using the $V-I$ color calibration given in Section 3.2); and the bottom right panel replaces the color-based extinction for the spectroscopic reddenings. The horizontal bars in each panel correspond to an adopted uncertainty of $187 \mathrm{~km} \mathrm{~s}^{-1}$ in the CMB redshift.

(A color version of this figure is available in the online journal.)

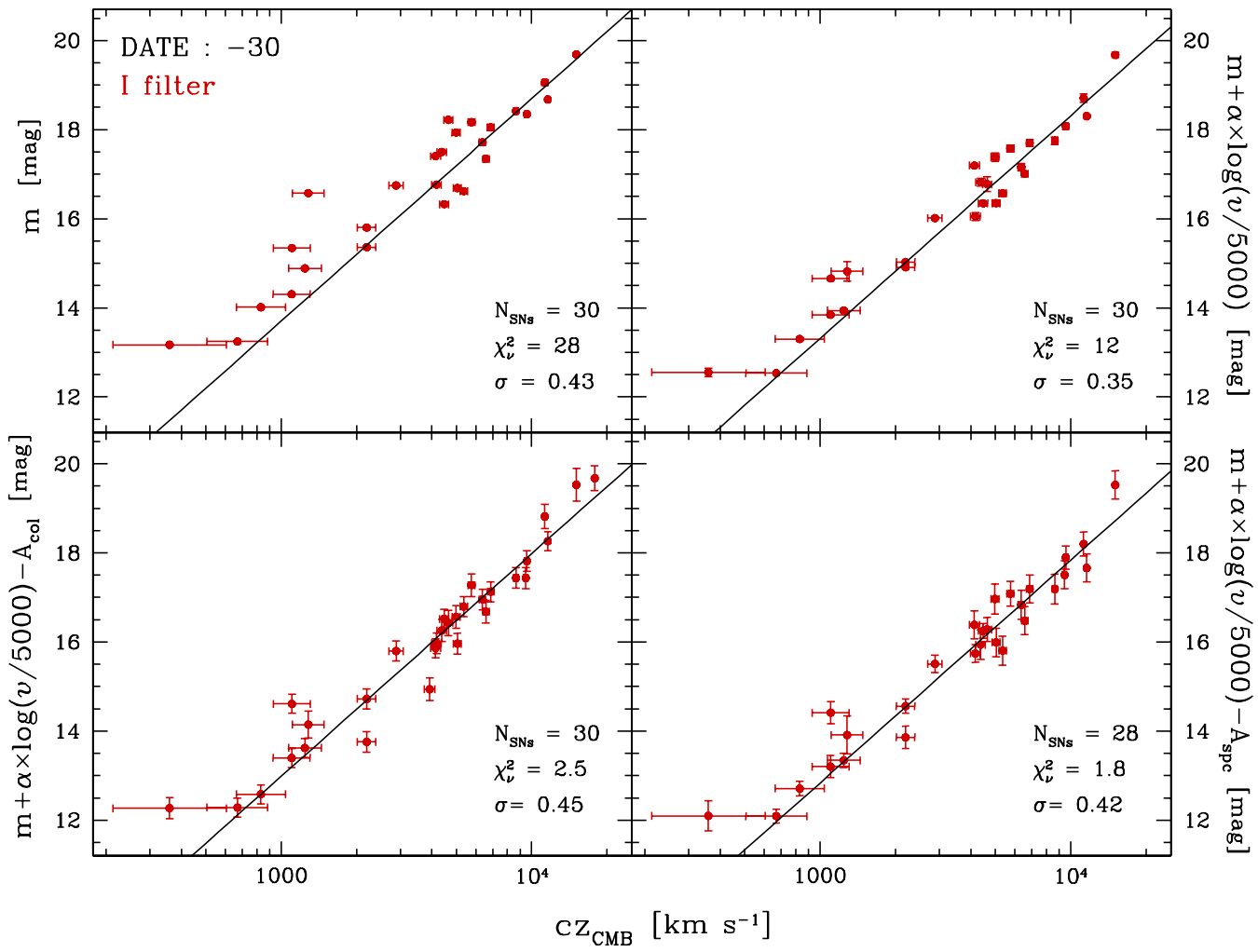

Figure 12. $I$-band HDs. The top left panel shows magnitudes interpolated to day -30 previously corrected for Galactic extinction and $K$-terms; the top right panel shows magnitudes additionally corrected for expansion velocities; the bottom left panel includes further corrections for color-based host-galaxy extinction (using the $V-I$ color calibration given in Section 3.2); and the bottom right panel replaces the color-based extinction for the spectroscopic reddenings. The horizontal bars in each panel correspond to an adopted uncertainty of $187 \mathrm{~km} \mathrm{~s}^{-1}$ in the CMB redshift.

(A color version of this figure is available in the online journal.) 
Table 7

$H_{0}$ Calculations

\begin{tabular}{|c|c|c|c|c|c|c|}
\hline \multirow[t]{2}{*}{$\mathrm{SN}$} & \multicolumn{3}{|c|}{$M+\alpha \log (v / 5000)-\beta(V-I)(\mathrm{mag})$} & \multicolumn{3}{|c|}{$H_{0}\left(\mathrm{~km} \mathrm{~s}^{-1} \mathrm{Mpc}^{-1}\right)$} \\
\hline & $B$ & $V$ & $I$ & $H_{0}(B)$ & $H_{0}(V)$ & $H_{0}(I)$ \\
\hline 1999em & $-17.94 \pm 0.20$ & $-18.41 \pm 0.20$ & $-18.24 \pm 0.19$ & $64.7 \pm 6.8$ & $62.2 \pm 6.1$ & $62.9 \pm 6.0$ \\
\hline Average & $-17.67 \pm 0.39$ & $-18.02 \pm 0.53$ & $-17.84 \pm 0.53$ & $71 \pm 12$ & $69 \pm 16$ & $\mathbf{7 0} \pm \mathbf{1 6}$ \\
\hline
\end{tabular}

Notes. The uncertainties in $\left\langle H_{0}\right\rangle$ and $\left\langle M_{\text {corr }}\right\rangle$ (the corrected absolute magnitude for $B V I$ in the second column) correspond to the weighted dispersion in each pair of values, which are a factor of 2-3 greater than the formal errors. This is a conservative uncertainty which takes into account the larger than expected discrepancy in absolute magnitude between the two objects.

\subsection{The Hubble Constant}

The Hubble constant is a parameter of central importance in cosmology which can be determined from our HDs. This can be accomplished as long as we can convert apparent magnitudes into distances. The calibration was done with two objects for which we found Cepheid distances in the literature: SN 1999em $(\mu=30.34 \pm 0.19$ mag; Leonard et al. 2002a) and SN 2004dj $(\mu=27.48 \pm 0.24 \mathrm{mag}$; Freedman et al. 2001). For each calibrating $\mathrm{SN}$ we can solve for $H_{0}$ using the expression

$$
\begin{aligned}
\log H_{0}= & 0.2\left[m+\alpha \log \left(v_{\mathrm{FeII}} / 5000\right)\right. \\
& -\beta(V-I)-\mu-z p+25]_{t=-30},
\end{aligned}
$$

where $m$ is the apparent magnitude, $v_{\text {FeII }}$ is the expansion velocity, and $V-I$ is the color of the calibrating $\mathrm{SN}$, all measured on day -30 and given in Table $5 ; \alpha, \beta$, and $z p$ are the fitting parameters given in the bottom three lines of Table 6 .

Table 7 summarizes our calculations for the two calibrators. We see that the corrected $B V I$ absolute magnitudes of $\mathrm{SN}$ 1999em and SN 2004dj differ from each other by 0.86$1.13 \mathrm{mag}$. This is considerably greater than the scatter of 0.3 mag in the LEV relation, but statistically not impossible; see Figure 15, where we plot with triangles the corrected absolute magnitudes of the two calibrating $\mathrm{SNe}$, on top of the whole sample of SNe employed in the HDs. This difference leads to $H_{0}$ values in the range $62-105 \mathrm{~km} \mathrm{~s}^{-1} \mathrm{Mpc}^{-1}$. With only two calibrating $\mathrm{SNe}$, the SCM still has much room for delivering a more accurate and precise value for $H_{0}$.

\subsection{Distances}

We are in a position to calculate distances to all of the $\mathrm{SNe}$ of our sample. This can be done with the expression

$$
\mu=m+\alpha \log \left(v_{\mathrm{FeII}} / 5000\right)-\beta(V-I)-\left\langle M_{\mathrm{corr}}\right\rangle,
$$

where $\left\langle M_{\text {corr }}\right\rangle$ is the corrected absolute magnitude of SNe IIP on day -30 . For this purpose, we employ the weighted mean of the two calibrating $\mathrm{SNe}$ given in Table 7. The resulting values are given in Table 8 for $32 \mathrm{SNe}$ IIP; the last column shows the weighted average of their distance moduli and its uncertainty, assuming no covariance. As Equation 16 shows, it is straightforward to shift the resulting distance moduli to accommodate a new value for $\left\langle M_{\text {corr }}\right\rangle$. Unfortunately, we are not able to estimate expansion velocities for five objects, and consequently not their distances as well.

As mentioned in Section 1, we can evaluate the accuracy of the SCM from a comparison with EPM distances. For this purpose, we employ EPM distances recently calculated by Jones et al. (2009), which are summarized in Table 9 along with our

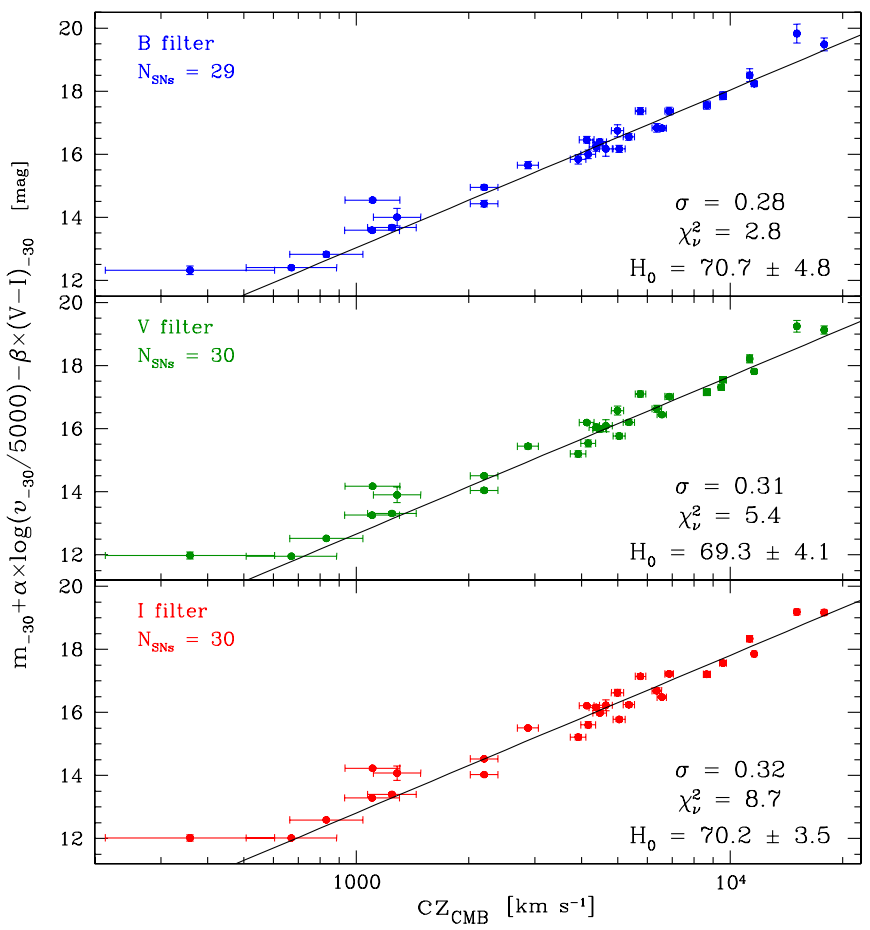

Figure 13. $B V I$ HDs leaving $R_{V}$ as a free parameter. In the top, middle, and bottom panels we show the HDs using $B V I$-band magnitudes of 29,30 , and $30 \mathrm{SNe}$, respectively. The horizontal bars in each panel correspond to an adopted uncertainty of $187 \mathrm{~km} \mathrm{~s}^{-1}$ in the CMB redshift.

(A color version of this figure is available in the online journal.)

distances for the 11 objects in common between SCM and EPM. Jones et al. (2009) determine EPM distances with two different sets of atmosphere models, both developed based on an analytic approach to calculate dilution factors as a function of a color temperature in order to correct the assumed blackbody emission. Their EPM distances were derived from average dilution factors and not from specific models for each SN. The EPM distances obtained using the models of Eastman et al. (1996, E96; Column 2 ) are $12 \% \pm 5 \%$ shorter than our SCM distances. On the other hand, the EPM distances determined using the models of Dessart \& Hillier (2005, D05; Column 3) are 40\% $\pm 10 \%$ greater than our SCM distances. Both percentage shifts are calculated weighted by the uncertainty in the EPM and SCM distances. These systematic differences can be seen in the top panel of Figure 17, and more clearly in the fractional differences $\left(d_{\mathrm{EPM}}-d_{\mathrm{SCM}}\right) / d_{\mathrm{SCM}}$ plotted in the bottom panel.

The systematic differences among the two sets of EPM distances can be solely attributed to the atmosphere models 
Table 8

Distance Moduli (mag)

\begin{tabular}{|c|c|c|c|c|c|}
\hline SN Name & Host Galaxy & $\mu_{B}$ & $\mu_{V}$ & $\mu_{I}$ & $\langle\mu\rangle$ \\
\hline 1991al & LEDA 140858 & $34.14(41)$ & $34.04(54)$ & $33.89(53)$ & $34.05(28)$ \\
\hline 1992af & ESO 340-G038 & $34.18(41)$ & $34.13(54)$ & $34.13(54)$ & $34.15(28)$ \\
\hline $1992 \mathrm{ba}$ & NGC 2082 & $31.34(40)$ & $31.31(54)$ & $31.34(53)$ & $31.33(28)$ \\
\hline $1999 b r$ & NGC 4900 & $31.67(48)$ & $31.89(59)$ & $32.10(57)$ & $31.86(31)$ \\
\hline $1999 \mathrm{cr}$ & ESO 576-G034 & $34.50(42)$ & $34.63(55)$ & $34.59(54)$ & $34.56(28)$ \\
\hline 1999em & NGC 1637 & $30.07(40)$ & $29.96(54)$ & $29.94(53)$ & $30.00(28)$ \\
\hline 1999gi & NGC 3184 & $30.48(40)$ & $30.51(54)$ & $30.52(53)$ & $30.50(28)$ \\
\hline 0210 & $\mathrm{MCG}+00-03-054$ & $37.52(50)$ & $37.27(57)$ & $37.09(54)$ & $37.31(31)$ \\
\hline $2002 \mathrm{fa}$ & NEAT J205221.51 & $37.23(44)$ & $37.16(55)$ & $37.03(54)$ & $37.16(29)$ \\
\hline $2002 \mathrm{gw}$ & NGC 922 & $33.35(41)$ & $33.46(54)$ & $33.44(53)$ & $33.41(28)$ \\
\hline 2002hj & NPM1G+04.0097 & $34.90(41)$ & $34.93(54)$ & $34.97(53)$ & $34.93(28)$ \\
\hline $2002 h x$ & PGC 23727 & $35.49(42)$ & $35.53(54)$ & $35.45(54)$ & $35.49(28)$ \\
\hline 2003B & NGC 1097 & $32.21(40)$ & $32.18(54)$ & $32.15(53)$ & $32.18(27)$ \\
\hline 2003E & MCG-4-12-004 & $33.91(42)$ & $34.04(54)$ & $34.10(54)$ & $34.01(28)$ \\
\hline $2003 \mathrm{~T}$ & UGC 4864 & $35.21(42)$ & $35.15(54)$ & $35.13(54)$ & $35.17(28)$ \\
\hline 2003bl & NGC 5374 & $33.95(45)$ & $34.09(57)$ & $34.23(55)$ & $34.07(30)$ \\
\hline $2003 \mathrm{bn}$ & 2MASX J10023529 & $33.67(42)$ & $33.54(55)$ & $33.53(54)$ & $33.60(28)$ \\
\hline $2003 \mathrm{ci}$ & UGC 6212 & $\ldots$ & $35.31(54)$ & $35.28(53)$ & $35.30(38)$ \\
\hline $2003 \mathrm{cn}$ & IC 849 & $34.77(42)$ & $34.86(55)$ & $34.85(54)$ & $34.81(28)$ \\
\hline $2003 c x$ & NEAT J135706.53 & $36.17(44)$ & $36.23(55)$ & $36.23(54)$ & $36.20(29)$ \\
\hline $2003 \mathrm{fb}$ & UGC 11522 & $34.41(44)$ & $34.56(55)$ & $34.55(54)$ & $34.49(29)$ \\
\hline $2003 \mathrm{gd}$ & M74 & $29.99(42)$ & $29.98(55)$ & $29.94(54)$ & $29.98(28)$ \\
\hline 2003hd & MCG-04-05-010 & $35.93(40)$ & $35.85(54)$ & $35.77(53)$ & $35.86(28)$ \\
\hline 2003hg & NGC 7771 & $33.50(42)$ & $33.18(54)$ & $33.14(54)$ & $33.31(28)$ \\
\hline $2003 \mathrm{hk}$ & NGC 1085 & $34.45(40)$ & $34.41(54)$ & $34.35(53)$ & $34.41(28)$ \\
\hline 2003hl & NGC 772 & $32.08(41)$ & $32.01(54)$ & $32.00(53)$ & $32.04(28)$ \\
\hline 2003hn & NGC 1448 & $31.14(40)$ & $31.15(54)$ & $31.11(53)$ & $31.13(27)$ \\
\hline 2003ho & ESO 235-G58 & $34.10(41)$ & $34.18(54)$ & $34.13(53)$ & $34.13(28)$ \\
\hline 2003ip & UGC 327 & $33.81(41)$ & $33.75(54)$ & $33.67(54)$ & $33.76(28)$ \\
\hline $2003 i q$ & NGC 772 & $32.50(40)$ & $32.40(54)$ & $32.34(53)$ & $32.43(28)$ \\
\hline $2004 d j$ & NGC 2403 & $28.06(43)$ & $28.22(55)$ & $28.19(54)$ & $28.14(29)$ \\
\hline 2004et & NGC 6946 & $28.64(50)$ & $28.21(58)$ & $28.17(55)$ & $28.37(31)$ \\
\hline
\end{tabular}

Notes. These values were calculated according to the approach discussed in Section 4.5. The mean value is a weighted average of the individual values for each filter.

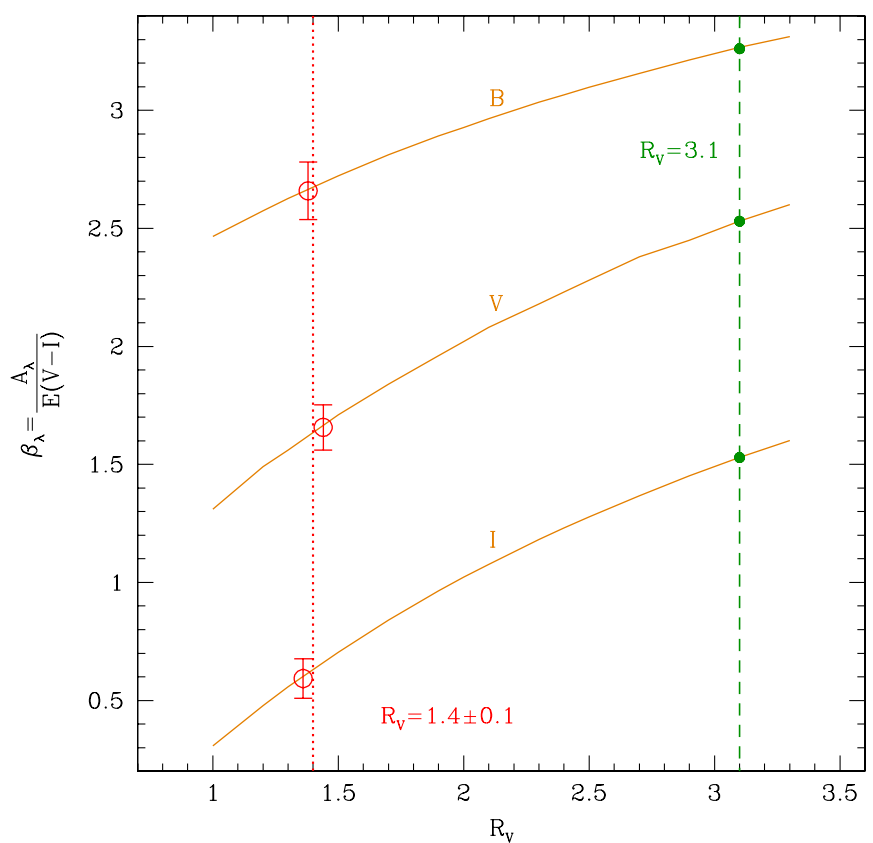

Figure 14. $R_{V}$ vs. $\beta$. The solid lines are computed from our library of SN II spectra for each of the $B V I$ bands. The $\beta$ parameters derived from the HDs in Figure 13 are shown with open circles for each of the $B V I$ bands. The dotted line shows the $R_{V}$-weighted mean for the $B V I$ values of $\beta$. The values for the standard Galactic extinction law are also shown with filled circles.
Table 9

EPM and SCM Distances

\begin{tabular}{lccc}
\hline \hline SN Name & $\begin{array}{c}d_{\mathrm{E} 96^{\mathrm{a}}} \\
(\mathrm{Mpc})\end{array}$ & $\begin{array}{c}d_{\mathrm{D} 05^{\mathrm{b}}} \\
(\mathrm{Mpc})\end{array}$ & $\begin{array}{c}d_{\mathrm{SCM}} \\
(\mathrm{Mpc})\end{array}$ \\
\hline $1992 \mathrm{ba}$ & $16.4(2.5)$ & $27.2(6.5)$ & $18.5(2.4)$ \\
$1999 \mathrm{br}$ & $\ldots$ & $39.5(13.5)$ & $23.6(3.4)$ \\
$1999 \mathrm{em}$ & $9.3(0.5)$ & $13.9(1.4)$ & $10.0(1.3)$ \\
$1999 \mathrm{gi}$ & $11.7(0.8)$ & $17.4(2.3)$ & $12.6(0.6)$ \\
$2002 \mathrm{gw}$ & $37.4(4.9)$ & $63.9(17.0)$ & $48.1(6.2)$ \\
$2003 \mathrm{~T}$ & $87.8(13.5)$ & $147.3(35.7)$ & $108.1(14.0)$ \\
$2003 \mathrm{bl}$ & $\ldots$ & $92.4(14.2)$ & $65.2(9.0)$ \\
$2003 \mathrm{bn}$ & $50.2(7.0)$ & $87.2(28.0)$ & $52.5(6.8)$ \\
$2003 \mathrm{hl}$ & $17.7(2.1)$ & $30.3(6.3)$ & $25.6(3.3)$ \\
$2003 \mathrm{hn}$ & $16.9(2.2)$ & $26.3(7.1)$ & $16.8(2.1)$ \\
$2003 \mathrm{iq}$ & $36.0(5.6)$ & $53.3(17.1)$ & $30.6(4.0)$ \\
\hline
\end{tabular}

Notes. Uncertainties are given in parentheses.

a EPM distances from atmosphere models by Eastman et al. (1996); see Jones et al. (2009).

b EPM distances from atmosphere models by Dessart \& Hillier (2005); see Jones et al. (2009). 


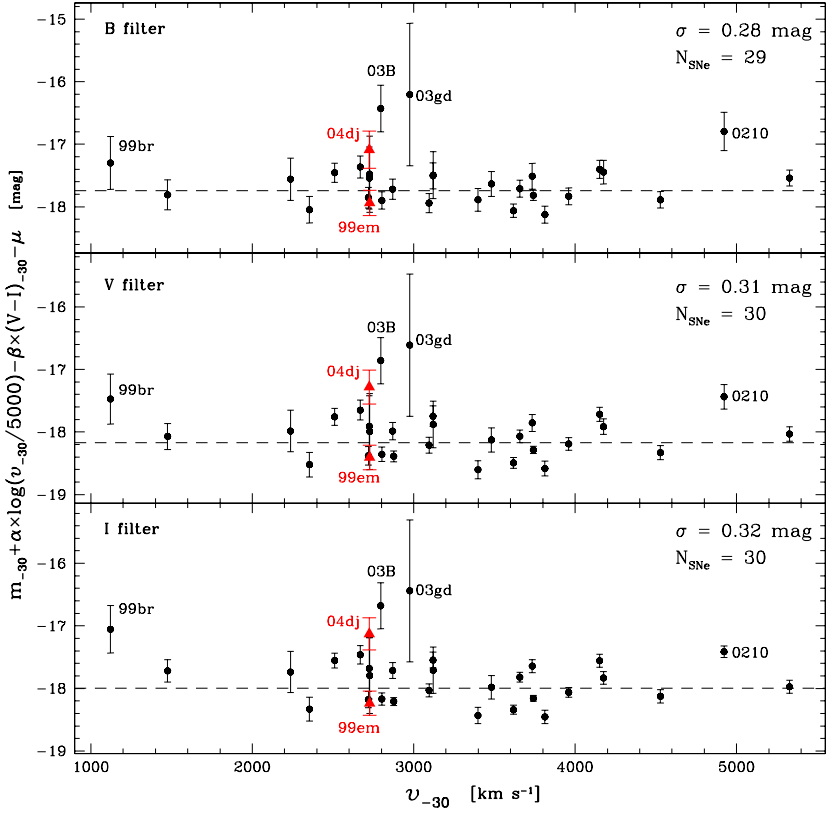

Figure 15. $B V I$-corrected absolute magnitudes (ordinate) against the Fe II expansion velocity (abscissa) for $29-30 \mathrm{SNe}$ (gray dots). We use the $H_{0}$ values of Table 7 and the CMB redshifts to compute the distance moduli. The dashed lines show the mean corrected absolute magnitude for the gray data points. The black triangles are the two calibrating $\mathrm{SNe}$, whose corrected absolute magnitudes were calculated using their Cepheid distances (see Table 7). Note that, as expected, the two calibrating $\mathrm{SNe}$ fall on each side of the corrected absolute magnitude distributions.

(A color version of this figure is available in the online journal.)

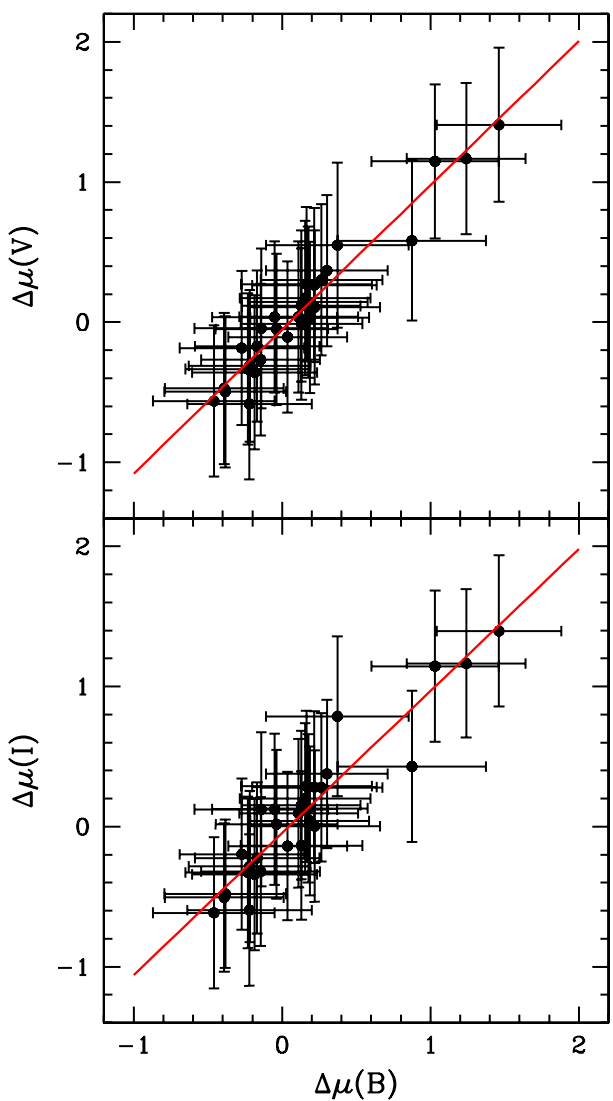

Figure 16. Residuals from the $B V I$ HDs. The correlation between the $V$-filter and $I$-filter residuals against the $B$-filter residuals is shown in the upper and lower panels, respectively. The gray lines represent the fit from which the scatter is measured.

(A color version of this figure is available in the online journal.)

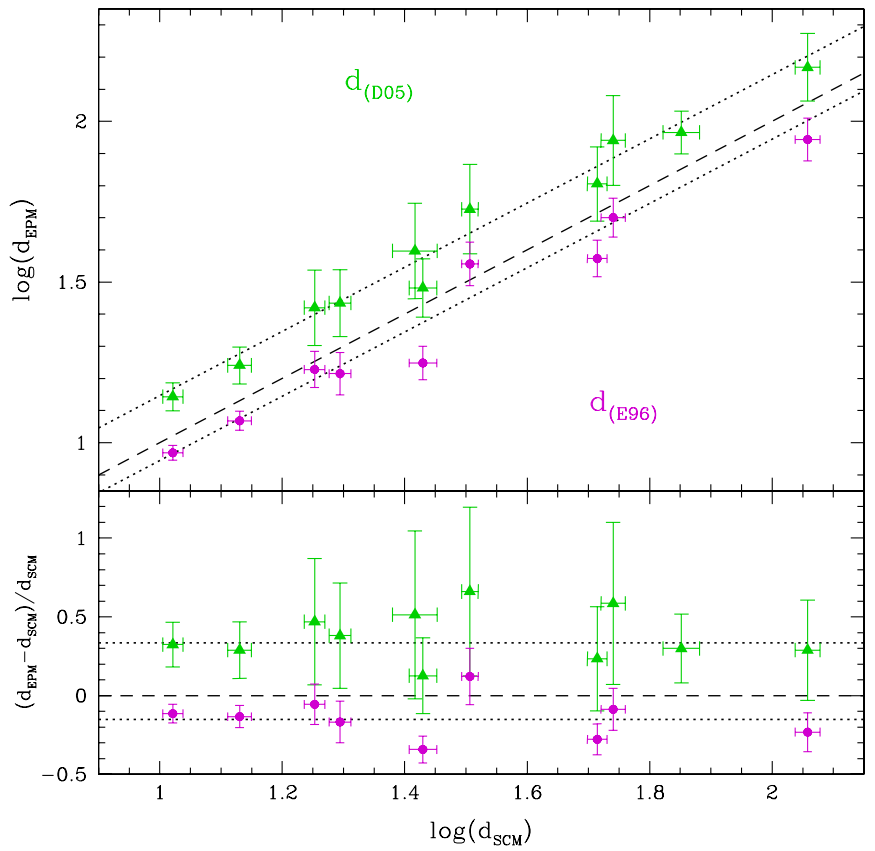

Figure 17. Direct comparison between SCM and EPM distances in Mpc calculated by Jones et al. (2009). Gray circles show the EPM distances computed from the atmosphere models of E96, while the black triangles represent EPM distances obtained from the D05 models. The bottom panel shows the fractional differences between both techniques against $\log \left(d_{\mathrm{SCM}}\right)$. In both panels, the dotted lines trace the systematic shifts of the EPM distances with respect to the SCM distances, $\sim 40 \%$ and $\sim 12 \%$, using D05 and E96 atmosphere models, respectively. The dashed line in the top panel is fixed to show a slope of unity. (A color version of this figure is available in the online journal.)

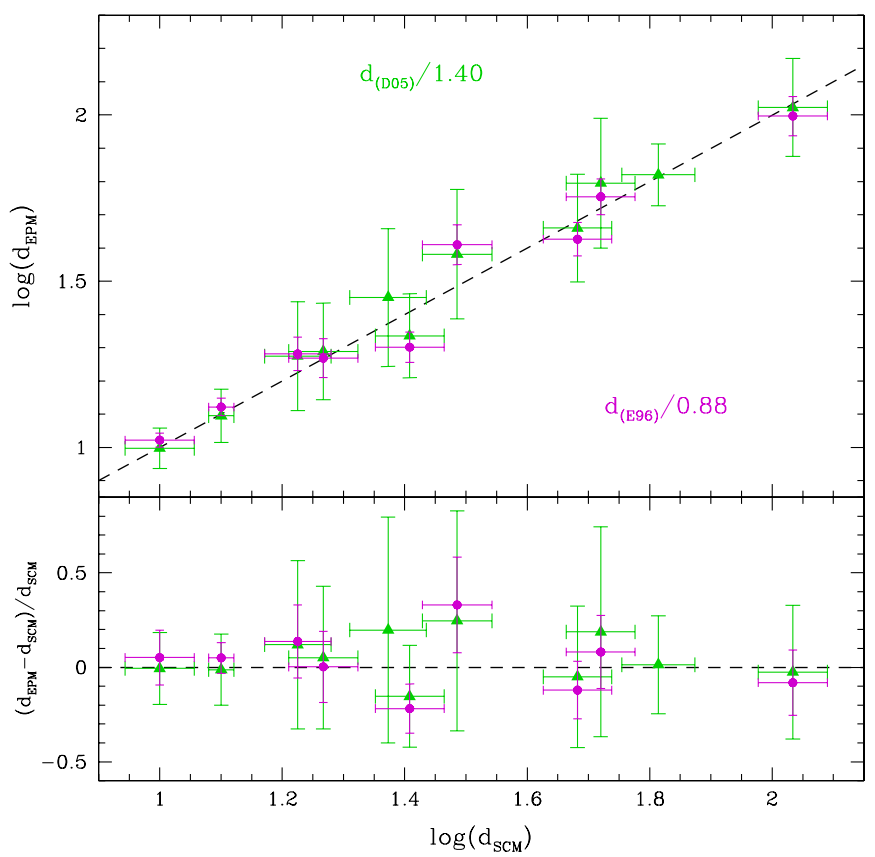

Figure 18. Similar to Figure 17, after removing systematic differences by bringing the EPM distances to the SCM scale (all in Mpc). Only random differences between EPM and SCM distances remain. For the E96 case, the discrepancies have a spread of $16 \%$, while for the D05 case the scatter is $13 \%$. The dashed line in the top panel is fixed to show a slope of unity.

(A color version of this figure is available in the online journal.) 
of E96 and D05. New radiative transfer models of SNe IIP are necessary to understand the origin of this discrepancy. Besides the systematic errors in both EPM implementations, we can gain an understanding of the internal precision of EPM and SCM after removing the systematic differences and bringing all distances to a common scale. For this purpose, we correct the EPM distances to the SCM distance scale by removing the percentage shifts. The comparison is shown in Figure 18; the distance differences have dispersions of $\sim 13 \%$ and $\sim 16 \%$ using D05 and E96, respectively. This implies that SCM and EPM produce relative distances with an internal precision $<13 \%-16 \%$. This agrees with the dispersions of $0.25-0.30$ mag seen in the HDs restricted to $\mathrm{SNe}$ with $c z_{\mathrm{CMB}}>3000 \mathrm{~km} \mathrm{~s}^{-1}$.

Further comparison between EPM and SCM can be made by taking the distance determination to SN 1999em. While Dessart $\&$ Hillier (2006) give a value of $11.5 \pm 1.0 \mathrm{Mpc}$, our SCM returns $12.6 \pm 0.6 \mathrm{Mpc}$. This agreement has a significance better than $1 \sigma$, taking into account both sources of error.

\section{DISCUSSION}

\subsection{Variations of the Extinction Law}

The reddening law that minimizes the dispersion in the $B V I$ HDs $\left(R_{V}=1.4 \pm 0.1\right)$ turns out to be very different from the standard Galactic extinction law $\left(R_{V}=3.1\right.$; Cardelli et al. 1989). Recently, Poznanski et al. (2009) carried out a similar analysis using SNe IIP, which confirmed the low value obtained for $R_{V}$ presented by Olivares (2008). There have also been several studies on this subject using SNe Ia. Most recently, Folatelli et al. (2010) solved for $R_{V}$ in the same manner as our approach (i.e., by minimizing the dispersion in the HD) and obtained a value of $R_{V} \approx 1.5$, remarkably consistent with our value. Further evidence for low $R_{\lambda}$ values using $\mathrm{SNe}$ Ia were reported by others applying a similar procedure (Phillips et al. 1999; Altavilla et al. 2004; Reindl et al. 2005; Hicken et al. 2009; Wang et al. 2009).

Other studies of dust reddening based on diverse methods were performed for nearby galaxies giving values for $R_{B}$ ranging between 2.4 and 4.3 (Rifatto 1990; Della Valle \& Panagia 1992; Brosch \& Loinger 1991; Finkelman et al. 2008). The ratio of total to selective absorption varies significantly over the range $R_{V} \approx 2.6-5.5$ even within our own Galaxy (Clayton \& Cardelli 1988).

A lower value of $R_{V}$ could be due to dust grains smaller than those in our Galaxy, since for a given value of $A_{V}$ the $E(B-V)$ reddening increases if the size of the grains decreases (Draine 2003). However, it would be very unlikely that our Galaxy is so special.

Most probably we are dealing with normal dust grains in the circumstellar medium, and therefore multiple scattering of photons makes the reddening law steeper and $R_{V}$ smaller (Wang 2005; Goobar 2008). This effect should be opposite in the ultraviolet, hence it could be further tested with photometry at such wavelengths.

\subsection{Internal Precision of the SCM}

The $B V I$ HDs have a scatter of $\sim 0.3 \mathrm{mag}$, implying a precision in individual distances of $\sim 15 \%$. Part of this scatter might be due to the peculiar velocities of the $\mathrm{SN}$ host galaxies, and the intrinsic precision of SCM could be even better. In fact, when we perform a comparison for $\mathrm{SNe}$ in common between SCM and EPM, the distance differences are 13\%-16\% (after removing the systematic difference among the SCM and EPM). This comparison is independent of the SN host-galaxy redshifts and implies that the internal precision in either of these two techniques must be lower than $13 \%-16 \%$, since these differences comprise the combined uncertainties of both methods. This is an encouraging result; it implies that both EPM and SCM can produce high-precision relative distances, thus offering a new route to cosmological parameters.

\subsection{The LEV Relation}

We note that the dispersion in the HDs increases with wavelength. This seems contrary to expectations given that (1) the extinction effects decrease with wavelength and (2) metallicity affects the $B$ band more than the other optical bands. Perhaps the luminosity is a function of not only velocity but also metallicity: $M_{V}=f(v,[\mathrm{Fe} / \mathrm{H}])$. If so, the velocity term in Equations (12) and (13) might be removing metallicity effects more efficiently at shorter wavelengths. This could be tested with $[\mathrm{Fe} / \mathrm{H}]$ measurements of SNe IIP. On the other hand, hydrodynamical simulations of SNe IIP suggest that there is an intrinsic scatter in the relation, which does not seem to be driven by any parameter of the model. Given the evidence we have, we can only claim a dependence of $L$ on $v$. The obvious physical explanation is that the internal energy is correlated with the kinetic energy, implying that the ratio of the internal energy to the kinetic energy is approximately independent of the explosion energy. As SN 1999br may suggest underluminous events might (1) change the shape of the LEV relation or (2) require a different explosion mechanism.

\section{CONCLUSIONS}

We established a library of 196 SN II optical spectra and developed a code which allows us to correct the observed photometry for Galactic extinction, $K$-terms, and host-galaxy extinction. We developed fitting procedures to the light curves, color curves, and velocity curves which allow us to precisely determine the transition time between the plateau and the tail phases. The use of this parameter as the time origin permitted us to line up the $\mathrm{SNe}$ to a common phase. The additional benefit of these fits is the interpolation of magnitudes, colors, and velocities over a wide range of epochs. The methodology explained above yields the following conclusions.

1. The comparison between our color-based dereddening technique and the spectroscopic reddenings is satisfactory within the uncertainties of both techniques. This is particularly encouraging since our method uses late-time photometric information, while the other method uses completely independent early-time spectroscopic data.

2. Using our new sample of SNe we recover the luminosity versus expansion-velocity trend (LEV relation) previously found by Hamuy \& Pinto (2002).

3. We construct $B V I$ HDs using two sets of host-galaxy reddenings. We demonstrate that the extinction corrections based on $V-I$ color do a better job than the spectroscopic determinations, reaching dispersions of $\sim 0.4 \mathrm{mag}$ in the HDs. This scatter is somewhat higher than the $0.35 \mathrm{mag}$ found previously by Hamuy $(2003 \mathrm{~b}, 2005)$. A much smaller dispersion of $0.3 \mathrm{mag}$ is achieved when we use $V-I$ colors to estimate reddening and allow $R_{V}$ to vary. We obtain $R_{V}=1.4 \pm 0.1$, much smaller than the Galactic value of 3.1. The low value of $R_{V}$ can be explained by a different nature of the dust grains in the host galaxies of SNe IIP or 
by scattering of light by circumstellar dust clouds (Wang 2005; Goobar 2008).

4. A clear correlation is found among residuals of BVI HDs. After removing the correlation, the rms in the HDs decreases to 6\%-9\% in distance, comparable to the precision achieved with SNe Ia.

5. After calibrating our HDs with Cepheid distances to $\mathrm{SN}$ 1999em and SN 2004dj, our BVI photometry leads to a Hubble constant in the range $62-105 \mathrm{~km} \mathrm{~s}^{-1} \mathrm{Mpc}^{-1}$; this agrees with most of the values obtained by means of diverse modern techniques.

6. Finally, we calculate the distance moduli to our SNe and make a comparison against EPM distances from Jones et al. (2009). The $11 \mathrm{SNe}$ in common show a systematic difference in distance between EPM and SCM, depending on the atmosphere models employed by EPM. Correcting for these shifts we bring the EPM distances to the SCM distance scale, from which we measure a dispersion of $13 \%-16 \%$. This spread reflects the combined internal precision of EPM and SCM. Therefore, the internal precision in either of these two techniques must be $<13 \%-16 \%$.

This analysis confirms the utility of SNe IIP as cosmological probes, providing strong encouragement to future high-redshift studies. We find that one can determine relative distances from SNe IIP with a precision of $15 \%$ or better. This uncertainty could be further reduced by including more SNe in the Hubble flow. In its current form the SCM requires both photometric and spectroscopic data. Since the latter are expensive to obtain (especially at high redshifts), it would be desirable to look for a photometric observable as a luminosity indicator instead of the expansion velocities.

We thank Luc Dessart, who generously provided the reddening estimates calculated by means of spectral fitting and the models plotted in Figure 4. F.O.E. also thanks him for stimulating discussions, as well as for accurate comments and suggestions. We are grateful to D. Leonard, J. Vinkó, D. Sahu, and A. Pastorello for providing digital spectra of SN 1999gi, SN 2004dj, SN 2004et, and SN 2005cs, respectively. F.O.E., M.H., G.P., and J.M. acknowledge support from the Millennium Center for Supernova Science through grant Pas06-045-F funded by "Programa Bicentenario de Ciencia y Tecnología de CONICYT" and "Programa Iniciativa Científica Milenio de MIDEPLAN," as well as support provided by Centro de Astrofísica FONDAP 15010003 and by Fondecyt through grant 1060808 from the Center of Excellence in Astrophysics and Associated Technologies (PFB 06). The supernova research of A.V.F.'s group at U.C. Berkeley has been financed by NSF grants AST-0607485 and AST-0908886, as well as by the TABASGO Foundation. Supernova research at the Harvard College Observatory has been supported by NSF grant AST-0606772. G.P. acknowledges partial support from Comité Mixto ESO-GOBIERNO DE CHILE. This paper is based in part on observations taken at the Cerro Tololo Inter-American Observatory, National Optical Astronomy Observatory, which is operated by the Association of Universities for Research in Astronomy, Inc. (AURA) under cooperative agreement with the NSF. Partly based on observations collected at the European Southern Observatory, Chile, in the course of program 163.H-0285.

Facilities: CTIO:0.9m (SITe No. 3 imaging CCD), CTIO:1.5m (CCD, CSPEC), YALO (ANDICAM, 2DF), Blanco (CSPEC, 2DF, CCD), Swope (SITe No. 3 imaging CCD), Du Pont (Tek No. 5 imaging CCD, WFCCD, MODSPEC,
2DF), Magellan:Baade (LDSS2 imaging spectrograph, Boller \& Chivens spectrograph), Magellan:Clay (LDSS2 imaging spectrograph), ESO:1.52m (IDS), Danish $1.54 \mathrm{~m}$ Telescope (DFOSC), Max Plank:2.2m (EFOSC2), NTT (EMMI), ESO:3.6m (EFOSC), SO:Kuiper (CCD), Bok (B\&C), Shane (Kast), FLWO:1.5m (FAST)

\section{REFERENCES}

Allen, S. W., Rapetti, D. A., Schmidt, R. W., Ebeling, H., Morris, R. G., \& Fabian, A. C. 2008, MNRAS, 383, 879

Altavilla, G., et al. 2004, MNRAS, 349, 1344

Anderson, J. P., \& James, P. A. 2008, MNRAS, 390, 1527

Arnett, D. 1996, Supernovae and Nucleosynthesis (Princeton, NJ: Princeton Univ. Press)

Astier, P., et al. 2006, A\&A, 447, 31

Baade, W., \& Zwicky, F. 1934, Phys. Rev., 46, 76

Barbon, R., Benetti, S., Rosino, L., Cappellaro, E., \& Turatto, M. 1990, A\&A, 237,79

Baron, E., Nugent, P. E., Branch, D., \& Hauschildt, P. H. 2004, ApJ, 616, L91

Bennett, C. L., et al. 2003, ApJS, 148, 1

Bersten, M. C., \& Hamuy, M. 2009, ApJ, 701, 200

Blake, C., \& Glazebrook, K. 2003, ApJ, 594, 665

Blondin, S., Prieto, J. L., Patat, F., Challis, P., Hicken, M., Kirshner, R. P., Matheson, T., \& Modjaz, M. 2009, ApJ, 693, 207

Botticella, M. T., et al. 2008, A\&A, 479, 49

Boughn, S., \& Crittenden, R. 2004, Nature, 427, 45

Brosch, N., \& Loinger, F. 1991, A\&A, 249, 327

Burrows, A. 2000, Nature, 403, 727

Capaccioli, M., Cappellaro, E., Mancini, D., \& Sedmak, G. 2003, Mem. Soc. Astron. Ital. Suppl., 3, 286

Cappellaro, E., Evans, R., \& Turatto, M. 1999, A\&A, 351, 459

Cardelli, J. A., Clayton, G. C., \& Mathis, J. S. 1989, ApJ, 345, 245

Castander, F. J. 2007, in ASP Conf. Ser. 379, Cosmic Frontiers, ed. N. Metcalfe \& T. Shanks (San Francisco, CA: ASP), 285

Clayton, G. C., \& Cardelli, J. A. 1988, AJ, 96, 695

Cousins, A. W. J. 1971, Royal Observatory Annals, 7 (Herstmonceux: Royal Greenwich Observatory), 86

D’Andrea, C. B., et al. 2010, ApJ, 708, 661

Della Valle, M., \& Panagia, N. 1992, AJ, 104, 696

Dessart, L., \& Hillier, D. J. 2005, A\&A, 439, 671

Dessart, L., \& Hillier, D. J. 2006, A\&A, 447, 691

Dessart, L., et al. 2008, ApJ, 675, 644

Draine, B. T. 2003, ARA\&A, 41, 241

Eastman, R. G., Schmidt, B. P., \& Kirshner, R. 1996, ApJ, 466, 911

Elmhamdi, A., Chugai, N. N., \& Danziger, I. J. 2003, A\&A, 404, 1077

Emerson, J. P., Sutherland, W. J., McPherson, A. M., Craig, S. C., Dalton, G. B., \& Ward, A. K. 2004, Messenger, 117, 27

Fabricant, D., Cheimets, P., Caldwell, N., \& Geary, J. 1998, PASP, 110, 79

Filippenko, A. V. 1982, PASP, 94, 715

Filippenko, A. V. 1997, ARA\&A, 35, 309

Finkelman, I., et al. 2008, MNRAS, 390, 969

Fixsen, D. J., Cheng, E. S., Gales, J. M., Mather, J. C., Shafer, R. A., \& Wright, E. L. 1996, ApJ, 473, 576

Folatelli, G., et al. 2010, AJ, 139, 120

Foley, R. J., et al. 2003, PASP, 115, 1220

Fosalba, P., et al. 2003, ApJ, 597, L89

Freedman, W. L., et al. 2001, ApJ, 553, 47

Freedman, W. L., et al. 2009, ApJ, 704, 1036

Frieman, J. A., Turner, M. S., \& Huterer, D. 2008, ARA\&A, 46, 385

Goobar, A. 2008, ApJ, 686, L103

Granlund, A., Conroy, P. G., Keller, S. C., Oates, A. P., Schmidt, B., Waterson, M. F., Kowald, E., \& Dawson, M. I. 2006, Proc. SPIE, 6269, 69

Hamuy, M. 2001, PhD thesis, Univ. Arizona

Hamuy, M. 2003a, arXiv:astro-ph/0301281

Hamuy, M. 2003b, arXiv:astro-ph/0309122

Hamuy, M. 2005, in Cosmic Explosions, ed. J. M. Marcaide \& K. W. Weiler (Berlin: Springer), 535

Hamuy, M., Phillips, M. M., Suntzeff, N. B., Schommer, R. A., Maza, J., \& Aviles, R. 1996, AJ, 112, 2391

Hamuy, M., \& Pinto, P. A. 2002, ApJ, 566, L63

Hamuy, M., Suntzeff, N. B., Bravo, J., \& Phillips, M. M. 1990, PASP, 102, 888

Hamuy, M., Suntzeff, N. B., Heathcote, S. R., Walker, A. R., Gigoux, P., \& Phillips, M. M. 1994, PASP, 106, 566 
Hamuy, M., Walker, A. R., Suntzeff, N. B., Gigoux, P., Heathcote, S. R., \& Phillips, M. M. 1992, PASP, 104, 533

Hamuy, M., et al. 2001, ApJ, 558, 615

Hendry, M. A., et al. 2005, MNRAS, 359, 906

Hicken, M., Wood-Vasey, W. M., Blondin, S., Challis, P., Jha, S., Kelly, P. L., Rest, A., \& Kirshner, R. P. 2009, ApJ, 700, 1097

Hobbs, L. M. 1974, ApJ, 191, 381

Hodapp, K. W., et al. 2004, Astron. Nachr., 325, 636

Hopkins, A. M., \& Beacom, J. F. 2006, ApJ, 651, 142

Janka, H.-T., Langanke, K., Marek, A., Martínez-Pinedo, G., \& Müller, B. 2007, Phys. Rep., 442, 38

Johnson, H. L., Iriarte, B., Mitchell, R. I., \& Wisniewski, W. Z. 1966, Commun. Lunar Planet. Lab., 4, 99

Jones, M. I., et al. 2009, ApJ, 696, 1176

Kasen, D., \& Woosley, S. E. 2009, ApJ, 703, 2205

Kirshner, R. P., \& Kwan, J. 1974, ApJ, 193, 27

Krisciunas, K., et al. 2009, AJ, 137, 34

Landolt, A. U. 1992, AJ, 104, 340

Law, N. M., et al. 2009, PASP, 121, 1395

Leonard, D. C., et al. 2002a, PASP, 114, 35

Leonard, D. C., et al. 2002b, AJ, 124, 2490

Matheson, T., et al. 2008, AJ, 135, 1598

Miller, J. S., \& Stone, R. P. S. 1993, Lick Obs. Tech. Rep. 66 (Santa Cruz, CA UCSC)

Minkowski, R. 1941, PASP, 53, 224

Munari, U., \& Zwitter, T. 1997, A\&A, 318, 269

Nadyozhin, D. K. 2003, MNRAS, 346, 97

Nelder, J. A., \& Mead, R. 1965, Comput. J., 7, 308

Nugent, P., et al. 2006, ApJ, 645, 841

Olivares E., F. 2008, MSc thesis, Univ. de Chile (arXiv:0810.5518)

Pastorello, A., et al. 2006, MNRAS, 370, 1752

Perlmutter, S., et al. 1999, ApJ, 517, 565

Phillips, M. M. 1993, ApJ, 413, L105

Phillips, M. M., Lira, P., Suntzeff, N. B., Schommer, R. A., Hamuy, M., \& Maza, J. 1999, AJ, 118, 1766
Poznanski, D., et al. 2009, ApJ, 694, 1067

Rau, A., et al. 2009, PASP, 121, 1334

Reindl, B., Tammann, G. A., Sandage, A., \& Saha, A. 2005, ApJ, 624, 532

Riess, A. G., Press, W. H., \& Kirshner, R. P. 1996, ApJ, 473, 88

Riess, A. G., et al. 1998, AJ, 116, 1009

Rifatto, A. 1990, in Proc. Fourth Int. Workshop of the Astronomical Observatory of Capodimonte, Dusty Objects in The Universe, ed. E. Bussoletti \& A. A. Vittone (Dordrecht: Kluwer), 277

Sahu, D. K., Anupama, G. C., Srividya, S., \& Muneer, S. 2006, MNRAS, 372 , 1315

Schlegel, D. J., Finkbeiner, D. P., \& Davis, M. 1998, ApJ, 500, 525

Schmidt, B. P., Kirshner, R. P., Eastman, R. G., Phillips, M. M., Suntzeff, N. B., Hamuy, M., Maza, J., \& Aviles, R. 1994, ApJ, 432, 42

Seo, H.-J., \& Eisenstein, D. J. 2003, ApJ, 598, 720

Spergel, D. N., et al. 2007, ApJS, 170, 377

Tonry, J. L., Blakeslee, J. P., Ajhar, E. A., \& Dressler, A. 2000, ApJ, 530, 625

Tonry, J. L., Dressler, A., Blakeslee, J. P., Ajhar, E. A., Fletcher, A. B., Luppino, G. A., Metzger, M. R., \& Moore, C. B. 2001, ApJ, 546, 681

Tripp, R. 1998, A\&A, 331, 815

Tsvetkov, D. Y., Volnova, A. A., Shulga, A. P., Korotkiy, S. A., Elmhamdi, A., Danziger, I. J., \& Ereshko, M. V. 2006, A\&A, 460, 769

Turatto, M., Benetti, S., \& Cappellaro, E. 2003, in Proc. ESO/MPA/MPE Workshop, From Twilight to Highlight: The Physics of Supernovae, ed. W. Hillebrandt \& B. Leibundgut (Berlin: Springer), 200

Tyson, J. A., Wittman, D. M., Hennawi, J. F., \& Spergel, D. N. 2003, Nucl. Phys. B, 124, 21

Utrobin, V. P. 2007, A\&A, 461, 233

Van Dyk, S. D., Li, W., \& Filippenko, A. V. 2003, PASP, 115, 1289

Vinkó, J., et al. 2006, MNRAS, 369, 1780

Wang, L. 2005, ApJ, 635, L33

Wang, X., et al. 2009, ApJ, 699, L139

Weaver, T. A., \& Woosley, S. E. 1980, in 9th Texas Symp. on Relativistic Astrophysics, ed. J. Ehlers, J. J. Perry, \& M. Walker (New York: New York Academy of Sciences), 335

Wood-Vasey, W. M., et al. 2007, ApJ, 666, 694 